\title{
DEGENERATE COMPLEX MONGE-AMPÈRE FLOWS ON STRICTLY PSEUDOCONVEX DOMAINS
}

\author{
DO HOANG SON
}

\begin{abstract}
We study the equation $\dot{u}=\log \operatorname{det}\left(u_{\alpha \bar{\beta}}\right)+f(t, z, u)$ in domains of $\mathbb{C}^{n}$. This equation has a close connection with the Kähler-Ricci flow. In this paper, we consider the case of the boundary conditions are smooth and the initial conditions are bounded.
\end{abstract}

\section{Contents}

Introduction

1. Strategy of the proof

2. Preliminaries

3. Order 1 a priori estimates

4. Higher order estimates

5. $C^{2, \alpha}$ estimate up to the boundary for the parabolic equation

6. Proof of the main theorem

7. Further directions

References

Date: June 26, 2018. 


\section{INTRODUCTION}

On Kähler manifolds, a Kähler-Ricci flow is an equation

$$
\frac{\partial}{\partial t} \omega=-\operatorname{Ric}(\omega)
$$

which starts from a Kähler metric. Here, $\operatorname{Ric}(\omega)$ is the form associated to the Ricci curvature of $\omega$, i.e., if

then

$$
\omega=\frac{\sqrt{-1}}{2 \pi} g_{i \bar{j}} d z^{i} \wedge \overline{d z^{j}}
$$

$$
\operatorname{Ric}(\omega)=-\frac{\sqrt{-1}}{2 \pi}\left(\partial_{i} \partial_{j} \log \operatorname{det} g\right) d z^{i} \wedge d \overline{z^{j}}
$$

This flow was become a poweful tool of geometry. The theory of Kähler-Ricci flow is well developed in the case of compact Kähler manifolds, see e.g. [Cao85, [PS05], [ST07, [Zha09], Tos10], GZ13], BG13]. It can be seen as the parabolic problem associated to an "elliptic" problem which would be the complex Monge-Ampère equation.

Monge-Ampère equations and their generalizations have long been studied in strictly pseudoconvex domains of $\mathbb{C}^{n}$, see for instance [CKNS85. This raises a natural question: what is the behavior of the corresponding parabolic equation in the case of $\mathbb{C}^{n}$ ?

Let $\Omega$ be a bounded smooth strictly pseudoconvex domain of $\mathbb{C}^{n}$, i.e., there exists a smooth strictly plurisubharmonic function $\rho$ defined on a bounded neighbourhood of $\bar{\Omega}$ such that $\Omega=\{\rho<0\}$ and $\left.d \rho\right|_{\partial \Omega} \neq 0$.

Let $T \in(0, \infty]$. We consider the equation

$$
\begin{cases}\dot{u}=\log \operatorname{det}\left(u_{\alpha \bar{\beta}}\right)+f(t, z, u) & \text { on } \Omega \times(0, T), \\ u=\varphi & \text { on } \partial \Omega \times[0, T), \\ u=u_{0} & \text { on } \bar{\Omega} \times\{0\},\end{cases}
$$

where $\dot{u}=\frac{\partial u}{\partial t}, u_{\alpha \bar{\beta}}=\frac{\partial^{2} u}{\partial z_{\alpha} \partial \bar{z}_{\beta}}, u_{0}$ is a plurisubharmonic function in a neighbourhood of $\Omega$ and $f$ is smooth in $[0, T) \times \bar{\Omega} \times \mathbb{R}$ and non increasing in the last variable.

This equation has a close connection with the Kähler-Ricci flow. There are some previous results. If $u_{0}$ is continuous and $\varphi$ does not depend on the last variable, then (2) admits a unique viscosity solution [EGZ14]. If $u_{0}$ is a smooth strictly plurisubharmonic function in $\bar{\Omega}, \varphi$ is smooth in $\bar{\Omega} \times[0, T)$ and the compatibility conditions are satisfied, then (2) admits a unique solution $u \in C^{\infty}(\Omega \times(0, T)) \cap C^{2 ; 1}(\bar{\Omega} \times[0, T))$ [HL10]; we state their result in detail as Theorem 2.2 in Section 2 .

In this paper, we study the case where $\varphi$ is smooth and $u_{0}$ is merely bounded. The main result is the following:

Theorem 0.1. Let $\Omega$ be a bounded smooth strictly pseudoconvex domain of $\mathbb{C}^{n}$ and $T \in(0, \infty]$. Let $u_{0}$ be a bounded plurisubharmonic function defined on a neighbourhood $\tilde{\Omega}$ of $\bar{\Omega}$. Assume that $\varphi \in C^{\infty}(\bar{\Omega} \times[0, T))$ and $f \in C^{\infty}([0, T) \times \bar{\Omega} \times \mathbb{R})$ satisfying

(i) $f_{u} \leq 0$.

(ii) $\varphi(z, 0)=u_{0}(z)$ for $z \in \partial \Omega$.

Then there exists a unique function $u \in C^{\infty}(\bar{\Omega} \times(0, T))$ such that

$$
u(., t) \text { is a strictly plurisubharmonic function on } \Omega \text { for all } t \in(0, T) \text {, }
$$




$$
\begin{gathered}
\dot{u}=\log \operatorname{det}\left(u_{\alpha \bar{\beta}}\right)+f(t, z, u) \text { on } \Omega \times(0, T), \\
u=\varphi \text { on } \partial \Omega \times(0, T), \\
\lim _{t \rightarrow 0} u(z, t)=u_{0}(z) \forall z \in \bar{\Omega} .
\end{gathered}
$$

Moreover, $u \in L^{\infty}\left(\bar{\Omega} \times\left[0, T^{\prime}\right)\right)$ for any $0<T^{\prime}<T$, and $u(., t)$ also converges to $u_{0}$ in capacity when $t \rightarrow 0$.

If $u_{0} \in C(\tilde{\Omega})$ then $u \in C(\bar{\Omega} \times[0, T))$.

Here, we say that $u(., t)$ converges to $u_{0}$ in capacity if the convergence is uniform outside sets of arbitrarily small capacity.

This improves the main result of [HL10] in two directions: we do not need smoothness of the initial data, and still have continuity when $t \rightarrow 0$; and we obtain the maximal possible regularity when $z$ tends to $\partial \Omega$, for fixed $t>0$.

Some techniques used in this paper are from the corresponding result in the case of compact Kähler manifolds. On a compact Kähler manifold, results have been obtained in the more general case where $u_{0}$ has zero or even positive Lelong numbers. We refer the reader to GZ13] and [DL14] for the details.

Acknowledgements. I am deeply grateful to Pascal Thomas and Vincent Guedj for many inspiring discussions on the subject and encouragement me to write down this paper. It is improved significantly thanks to their thorough reading and editing. I also would like to thank Lu Hoang Chinh for very useful discussions about Proposition 3.3.

\section{Strategy of the PRoOF}

We fix some notation. We say that $u \in C^{2 ; 1}(\bar{\Omega} \times[0, T))$ if $u(., t) \in C^{2}(\bar{\Omega})$ for any $t \in[0, T), u(z,.) \in C^{1}([0, T))$ for any $z \in \bar{\Omega}$ and $\dot{u}, u_{s_{j} s_{k}} \in C(\bar{\Omega} \times[0, T))$ for $s_{j}, s_{k} \in\left\{x_{1}, y_{1}, \ldots x_{n}, y_{n}\right\}$.

In order to prove Theorem 0.1, we use an approximation process and we first will need to prove the following a priori estimates theorem:

Theorem 1.1. Let $\Omega$ be a bounded smooth strictly pseudoconvex domain of $\mathbb{C}^{n}$ and $T>0$. Let $\varphi \in C^{\infty}(\bar{\Omega} \times[0, T))$ and $f \in C^{\infty}([0, T) \times \bar{\Omega} \times \mathbb{R})$ and let $u \in C^{\infty}(\Omega \times$ $(0, T)) \cap C^{2 ; 1}(\bar{\Omega} \times[0, T))$, strictly plurisubharmonic with respect to $z$, be a solution of the equation

$$
\dot{u}=\log \operatorname{det}\left(u_{\alpha \bar{\beta}}\right)+f(t, z, u) \quad \text { on } \Omega \times(0, T) \text {. }
$$

Assume that

$$
\begin{gathered}
\left.u\right|_{\partial \Omega \times[0, T)}=\left.\varphi\right|_{\partial \Omega \times[0, T)}, \\
\sup |u(z, 0)| \leq C_{u}, \\
f_{u}(t, z, u) \leq 0 \quad \forall(t, z, u) \in(0, T) \times \Omega \times \mathbb{R}, \\
\|f\|_{C^{2}((0, T) \times \Omega \times \mathbb{R})} \leq C_{f}, \\
\|\varphi\|_{C^{4}(\Omega \times(0, T))} \leq C_{\varphi} .
\end{gathered}
$$


Then there exists $M_{0}=M_{0}\left(\Omega, T, C_{u}, C_{\varphi}, C_{f}\right)$ and for any $0<\epsilon<T$ there exists $C=C\left(\Omega, \epsilon, T, C_{u}, C_{\varphi}, C_{f}\right)$ such that

$$
\begin{gathered}
|u| \leq M_{0} \quad \text { on } \quad \Omega \times(0, T), \\
|\nabla u|+|\dot{u}|+\Delta u \leq C \quad \text { on } \quad \Omega \times(\epsilon, T) .
\end{gathered}
$$

Remark 1.2. In the theorem above, we denote

$$
\begin{aligned}
\|\varphi\|_{C^{k}(\Omega \times(0, T))} & =\sum_{|j|+2 l \leq k} \sup _{\Omega \times(0, T)}\left|D_{s}^{j} D_{t}^{l} \varphi\right|, \\
\|f\|_{\left.C^{k}((0, T) \times \Omega \times \mathbb{R})\right)} & =\sum_{j_{1}+\left|j_{2}\right|+j_{3} \leq k} \sup _{t}\left|D_{t}^{j_{1}} D_{s}^{j_{2}} D_{u}^{j_{3}} f\right|,
\end{aligned}
$$

where $s=\left(s_{1}, \ldots, s_{2 n}\right)=\left(x_{1}, y_{1}, \ldots, x_{n}, y_{n}\right)$.

For the proof of Theorem 0.1 , the strategy is as follows.

+ Construct the solutions $u_{m} \in C^{\infty}(\Omega \times(0, T)) \cap C^{2 ; 1}(\bar{\Omega} \times[0, T))$ of (4) such that $\left.u_{m}\right|_{\bar{\Omega} \times\{0\}}$ and $\left.u_{m}\right|_{\partial \Omega \times(0, T)}$ converge pointwise, respectively, to $u_{0}$ and $\left.\varphi\right|_{\partial \Omega \times(0, T)}$. We also ask that the $u_{m}$ be uniformly bounded and $\left.u_{m}\right|_{\partial \Omega \times\left(\epsilon_{m}, T\right)}=\left.\varphi\right|_{\partial \Omega \times\left(\epsilon_{m}, T\right)}$ for some $\epsilon_{m} \searrow 0$.

+ Use the a priori estimates to prove

$$
\left\|u_{m}\right\|_{C^{2}\left(\bar{\Omega} \times\left(\epsilon, T^{\prime}\right)\right)} \leq C_{\epsilon, T^{\prime}}
$$

for any $0<\epsilon<T^{\prime}<T$, where $C_{\epsilon, T^{\prime}}>0$ is independent of $m$.

+ Use $C^{2, \alpha}$ estimates and to prove

$$
\left\|u_{m}\right\|_{C^{k}\left(\bar{\Omega} \times\left(\epsilon, T^{\prime}\right)\right)} \leq C_{k, \epsilon, T^{\prime}}
$$

for any $0<\epsilon<T^{\prime}<T$ and $k>0$, where $C_{k, \epsilon, T^{\prime}}>0$ is independent on $m$. The $C^{2, \alpha}$ estimates and the $C^{k, \alpha}$ regularity will be mentioned in section 5 .

+ By Ascoli's theorem, there exists a subsequence of $\left\{u_{m}\right\}$, denoted also by $\left\{u_{m}\right\}$, and $u \in C^{\infty}(\bar{\Omega} \times(0, T)$ such that

$$
u_{m} \stackrel{C^{k}\left(\bar{\Omega} \times\left(\epsilon, T^{\prime}\right)\right)}{\longrightarrow} u .
$$

Then, $u$ satisfies (3), (44) and (15).

+ Use Comparison principle to prove (6).

+ Finally, we prove the uniqueness of $u$.

We will study some important tools before we prove Theorem 0.1 . In Section 2, we introduce some basic results about parabolic complex Monge-Ampère equations. In Sections 3 and 4, we prove the a priori estimates theorem (Theorem 1.1). In Section 5 we establish the $C^{2, \alpha}$ estimate needed to solve our problem. Finally in Section [6] we prove Theorem 0.1 .

\section{Preliminaries}

\subsection{Hou-Li theorem.}

The Hou-Li theorem states that equation (2) has a unique solution when the conditions are good enough. We will use it in Section 6 to obtain smooth solutions to an approximating problem, to which we then will apply the a priori estimates from Theorem 1.1. 
We first need the notion of subsolution.

Definition 2.1. A function $\underline{u} \in C^{\infty}(\bar{\Omega} \times[0, T))$ is called a subsolution of the equation (14) if and only if

$$
\left\{\begin{array}{l}
\underline{u}(., t) \text { is a strictly plurisubharmonic function, } \\
\underline{u} \leq \log \operatorname{det}(\underline{u})_{\alpha \bar{\beta}}+f(t, z, \underline{u}), \\
\left.\underline{u}\right|_{\partial \Omega \times(0, T)}=\left.\varphi\right|_{\partial \Omega \times(0, T)} \\
\underline{u}(., 0) \leq u_{0} .
\end{array}\right.
$$

Theorem 2.2. Let $\Omega \subset \mathbb{C}^{n}$ be a bounded domain with smooth boundary. Let $T \in$ $(0, \infty]$. Assume that

- $\varphi$ is a smooth function in $\bar{\Omega} \times[0, T)$.

- $f$ is a smooth function in $[0, T) \times \bar{\Omega} \times \mathbb{R}$ non increasing in the lastest variable.

- $u_{0}$ is a smooth strictly plurisubharmonic funtion in a neighborhood of $\Omega$.

- $u_{0}(z)=\varphi(z, 0), \forall z \in \partial \Omega$.

- The compatibility condition is satisfied, i.e.

$$
\dot{\varphi}=\log \operatorname{det}\left(u_{0}\right)_{\alpha \bar{\beta}}+f\left(t, z, u_{0}\right), \quad \forall(z, t) \in \partial \Omega \times\{0\} .
$$

- There exists a subsolution to the equation (14).

Then there exists a unique solution $u \in C^{\infty}(\Omega \times(0, T)) \cap C^{2 ; 1}(\bar{\Omega} \times[0, T))$ of the equation

$$
\begin{cases}\dot{u}=\log \operatorname{det}\left(u_{\alpha \bar{\beta}}\right)+f(t, z, u) & \text { on } \Omega \times(0, T), \\ u=\varphi & \text { on } \partial \Omega \times[0, T), \\ u=u_{0} & \text { on } \bar{\Omega} \times\{0\} .\end{cases}
$$

Remark 2.3. (i) There is a corresponding result in the case of a compact Kähler manifold. On the compact Kähler manifold $X$, we must assume that $0<T<$ $T_{\max }$, where $T_{\max }$ depends on $X$. In the case of domain $\Omega \subset \mathbb{C}^{n}$, we can assume that $T=+\infty$ if $\varphi, \underline{u}$ are defined on $\bar{\Omega} \times[0,+\infty)$ and $f$ is defined on $[0,+\infty) \times \bar{\Omega} \times \mathbb{R}$.

(ii) If $\Omega$ is a bounded smooth strictly pseudoconvex domain of $\mathbb{C}^{n}$ then one can prove that a subsolution always exists, and so Theorem 2.2 does not need the additional assumpation of existence of a subsolution.

\subsection{Maximum principle.}

The following maximum principle is a basic tool to establish upper and lower bounds in the sequel (see [BG13] and [IS13] for the proof).

Theorem 2.4. Let $\Omega$ be a bounded domain of $C^{n}$ and $T>0$. Let $\left\{\omega_{t}\right\}_{0<t<T}$ be a continuous family of continuous positive definite Hermitian forms on $\Omega$. Denote by $\Delta_{t}$ the Laplacian with respect to $\omega_{t}$ :

$$
\Delta_{t} f=\frac{n \omega_{t}^{n-1} \wedge d d^{c} f}{\omega_{t}^{n}}, \forall f \in C^{\infty}(\Omega) .
$$

Suppose that $H \in C^{\infty}(\Omega \times(0, T)) \cap C(\bar{\Omega} \times[0, T))$ and satisfies 


$$
\left(\frac{\partial}{\partial t}-\Delta_{t}\right) H \leq 0 \text { or } \dot{H}_{t} \leq \log \frac{\left(\omega_{t}+d d^{c} H_{t}\right)^{n}}{\omega_{t}^{n}} .
$$

Then $\sup _{\bar{\Omega} \times[0, T)} H=\sup _{\partial_{P}(\Omega \times[0, T))} H$. Here we denote $\partial_{P}(\Omega \times(0, T))=\partial \Omega \times(0, T) \cup \bar{\Omega} \times\{0\}$.

Corollary 2.5. (Comparison principle) Let $\Omega$ be a bounded domain of $\mathbb{C}^{n}$ and $T \in$ $(0, \infty]$. Let $u, v \in C^{\infty}(\Omega \times(0, T)) \cap C(\bar{\Omega} \times[0, T))$ satisfying

- $u(., t)$ and $v(., t)$ are strictly plurisubharmonic functions for any $t \in[0, T)$,

- $\dot{u} \leq \log \operatorname{det}\left(u_{\alpha \bar{\beta}}\right)+f(t, z, u)$,

- $\dot{v} \geq \log \operatorname{det}\left(v_{\alpha \bar{\beta}}\right)+f(t, z, v)$,

where $f \in C^{\infty}([0, T) \times \bar{\Omega} \times \mathbb{R})$ is non increasing in the last variable.

Then $\sup _{\Omega \times(0, T)}(u-v) \leq \max \left\{0, \sup _{\partial_{P}(\Omega \times(0, T))}(u-v)\right\}$.

Corollary 2.6. Let $\Omega$ be a bounded domain of $\mathbb{C}^{n}$ and $T \in(0, \infty]$. We denote by $L$ a operator on $C^{\infty}(\Omega \times(0, T))$ satisfying

$$
L(f)=\frac{\partial f}{\partial t}-\sum a_{\alpha \bar{\beta}} \frac{\partial^{2} f}{\partial z_{\alpha} \partial \bar{z}_{\beta}}-b . f,
$$

where $a_{\alpha \bar{\beta}}, b \in C(\Omega \times(0, T)),\left(a_{\alpha \bar{\beta}}(z, t)\right)$ are positive definite Hermitian matrices and $b(z, t)<0$.

Assume that $\phi \in C^{\infty}(\Omega \times(0, T)) \cap C(\bar{\Omega} \times[0, T))$ satisfies

$$
L(\phi) \leq 0
$$

Then $\phi \leq \max \left(0, \sup _{\partial_{P}(\Omega \times(0, T))} \phi\right)$.

\subsection{The Laplacian inequalities.}

We shall need two standard auxiliary results (see [Yau78], [Siu87] for a proof).

Theorem 2.7. Let $\omega_{1}, \omega_{2}$ be positive $(1,1)$-forms on a complex manifold $X$. Then

$$
n\left(\frac{\omega_{1}^{n}}{\omega_{2}^{n}}\right)^{1 / n} \leq \operatorname{tr}_{\omega_{2}}\left(\omega_{1}\right) \leq n\left(\frac{\omega_{1}^{n}}{\omega_{2}^{n}}\right)\left(\operatorname{tr}_{\omega_{1}}\left(\omega_{2}\right)\right)^{n-1},
$$

where $\operatorname{tr}_{\omega_{1}}\left(\omega_{2}\right)=\frac{n \omega_{1}^{n-1} \wedge \omega_{2}}{\omega_{1}^{n}}$.

Theorem 2.8. Let $\omega, \omega^{\prime}$ be two Kähler forms on a complex manifold $X$. If the holomorphic bisectional curvature of $\omega$ is bounded below by a constant $B \in \mathbb{R}$ on $X$, then

$$
\Delta_{\omega^{\prime}} \log \operatorname{tr}_{\omega}\left(\omega^{\prime}\right) \geq-\frac{\operatorname{tr}_{\omega} \operatorname{Ric}\left(\omega^{\prime}\right)}{\operatorname{tr} \omega\left(\omega^{\prime}\right)}+B \operatorname{tr}_{\omega^{\prime}}(\omega)
$$

where Ric $\left(\omega^{\prime}\right)$ is the form associated to the Ricci curvature of $\omega^{\prime}$.

Remark 2.9. Applying Theorem 2.8 for $\omega=d d^{c}|z|^{2}$ and $\omega^{\prime}=d d^{c} u$, we have

$$
\sum u^{\alpha \bar{\beta}}(\log \Delta u)_{\alpha \bar{\beta}} \geq \frac{\Delta \log \operatorname{det}\left(u_{\alpha \bar{\beta}}\right)}{\Delta u} .
$$




\subsection{Construction of subsolutions.}

We give a first construction which will be used in the proof of Theorem 1.1. First we need a notion of subsolution weaker than the one in Definition 2.1.

Definition 2.10. We say that a function $\underline{u} \in C^{\infty}(\bar{\Omega} \times[0, T))$ is a subsolution of the equation (7) if

$$
\underline{\dot{u}} \leq \log \operatorname{det}\left(\underline{u}_{\alpha \bar{\beta}}\right)+f(t, z, \underline{u}) .
$$

We will construct subsolutions of (7) in order to prove some estimates on the boundary.

Let $\rho \in S P S H(\bar{\Omega}) \cap C^{\infty}(\bar{\Omega})$ be a function which defines $\Omega$. We also assume that $\inf \rho=-1$. Let $\zeta \in C^{\infty}(\mathbb{R})$ such that $0 \leq \zeta \leq 1,\left.\zeta\right|_{[0,1]}=1$ and $\left.\zeta\right|_{[2, \infty)}=0$.

Let $\varphi$ and $u_{0}$ be as in Theorem 1.1. For any $m>0$, we denote the function $\varphi_{m} \in$ $C^{\infty}(\bar{\Omega} \times[0, T))$ by the formula

$$
\varphi_{m}=\varphi-O s c\left(u_{0}\right) \cdot \zeta(m t) .
$$

Then there exists $M_{m}>0$ depending on $\rho, T, C_{u}, C_{\varphi}, C_{f}$ such that the function $\underline{u}_{m}=$ $\varphi_{m}+M_{m} \rho$ satisfies

$$
\begin{gathered}
\underline{\dot{u}}_{m} \leq \log \operatorname{det}\left(\underline{u}_{m}\right)_{\alpha \bar{\beta}}+f\left(t, z, \underline{u}_{m}\right) \text { on } \Omega \times(0, T), \\
d d^{c}\left(\underline{u}_{m}\right) \geq d d^{c}|z|^{2} \text { on } \Omega \times[0, T) .
\end{gathered}
$$

Then $\underline{u}_{m}$ is a subsolution of (17). Moreover,

$$
\begin{aligned}
\left.\underline{u}_{m}\right|_{\partial_{P}(\Omega \times(0, T))} & \leq\left. u\right|_{\partial_{P}(\Omega \times(0, T))}, \\
\left.\underline{u}_{m}\right|_{\partial \Omega \times\left(\frac{2}{m}, T\right)} & =\left.\varphi\right|_{\partial \Omega \times\left(\frac{2}{m}, T\right)} .
\end{aligned}
$$

By the maximum principle, we have

$$
\underline{u}_{m} \leq u \text { on } \Omega \times(0, T) .
$$

In the next two sections, we will prove Theorem 1.1. For convenience, we define an operator $L$ on $C^{\infty}(\Omega \times(0, T))$ by the formula

$$
L(\phi)=\dot{\phi}-\sum u^{\alpha \bar{\beta}} \phi_{\alpha \bar{\beta}}-f_{u}(t, z, u) \phi,
$$

where $u$ is the function in Theorem 1.1 and $\left(u^{\alpha \bar{\beta}}\right)$ is the transpose of inverse matrix of Hessian matrix $\left(u_{\alpha \bar{\beta}}\right)$.

\section{ORDER 1 A PRIORI ESTIMATES}

In this section, we will estimate $u, \dot{u}$ and $|\nabla u|$. Clearly,

$$
\underline{u}_{1} \leq u \leq \sup _{\partial \Omega \times(0, T)} \varphi \text { on } \Omega \times(0, T) .
$$

Then

$$
-M_{1}-2 \sup |\varphi|-C_{u} \leq u(z, t) \leq \sup _{\partial \Omega \times(0, T)} \varphi
$$

where $M_{1}$ is the constant defined in 2.4. Let $C_{1}=M_{1}+2 C_{\varphi}+C_{u}$, we obtain

$$
\sup |u| \leq C_{1} \text {. }
$$




\subsection{Bounds on $\dot{u}$.}

Proposition 3.1. There exists $C_{2}>0$ depending only on $T, C_{f}, C_{1}$ such that

$$
t|\dot{u}| \leq C_{2} \text { on } \Omega \times(0, T) .
$$

Proof. Take $L$ as in (15), then

$$
L(t \dot{u}-u)=t \ddot{u}-t \sum u^{\alpha \bar{\beta}} \dot{u}_{\alpha \bar{\beta}}+n-(t \dot{u}-u) f_{u}(t, z, u) .
$$

By equation (7), we have

$$
t \ddot{u}=t \sum u^{\alpha \bar{\beta}} \dot{u}_{\alpha \bar{\beta}}+t . f_{t}(t, z, u)+t \dot{u} \cdot f_{u}(t, z, u) .
$$

Then

$$
-C_{2}^{\prime} \leq L(t \dot{u}-u)=n+t . f_{t}(t, z, u)+u \cdot f_{u}(t, z, u) \leq C_{2}^{\prime},
$$

where $C_{2}^{\prime}=n+C_{f}\left(T+C_{1}\right)>0$.

Since $L\left(t \dot{u}-u-C_{2}^{\prime} t\right) \leq 0$ and $L\left(t \dot{u}-u+C_{2}^{\prime} t\right) \geq 0$, by the maximum principle, we obtain

$$
\begin{gathered}
t \dot{u}-u-C_{2}^{\prime} t \leq \sup _{\partial_{P}(\Omega \times(0, T))}\left(t \dot{u}-u-C_{2}^{\prime} t\right) \leq\left(C_{\varphi}+C_{2}^{\prime}\right) T+C_{1}, \\
t \dot{u}-u+C_{2}^{\prime} t \geq \inf _{\partial_{P}(\Omega \times(0, T))}\left(t \dot{u}-u+C_{2}^{\prime} t\right) \geq-\left(C_{\varphi}+C_{2}^{\prime}\right) T-C_{1} .
\end{gathered}
$$

Thus $t|\dot{u}| \leq C_{2}$ on $\Omega \times(0, T)$, where $C_{2}=\left(C_{\varphi}+2 C_{2}^{\prime}\right) T+2 C_{1}$.

\subsection{Gradient estimates.}

Proposition 3.2. Let $m>\frac{2}{T}$. Then there exists $C_{3}=C_{3}\left(\Omega, M_{m}, C_{\varphi}\right)>0$ such that

$$
|\nabla u| \leq C_{3} \text { on } \partial \Omega \times\left(\frac{2}{m}, T\right) .
$$

Proof. Let $h \in C^{\infty}(\bar{\Omega} \times[0, T))$ be a spatial harmonic function (i.e. harmonic with respect to $z$ ) satisfying

$$
h=\varphi \text { on } \partial \Omega \times[0, T) .
$$

Then taking $\underline{u}_{m}$ as 2.4 , we have

$$
\begin{gathered}
\underline{u}_{m} \leq u \leq h \text { on } \Omega \times\left(\frac{2}{m}, T\right), \\
\underline{u}_{m}=u=h=\varphi \text { on } \partial \Omega \times\left(\frac{2}{m}, T\right) .
\end{gathered}
$$

Hence

$$
\left|\nabla\left(u-\underline{u}_{m}\right)\right| \leq\left|\nabla\left(h-\underline{u}_{m}\right)\right| \text { on } \partial \Omega \times\left(\frac{2}{m}, T\right) .
$$

Thus

$$
|\nabla u| \leq\left|\nabla \underline{u}_{m}\right|+\left|\nabla\left(h-\underline{u}_{m}\right)\right| \leq C_{3} \text { on } \partial \Omega \times\left(\frac{2}{m}, T\right),
$$

where $C_{3}>0$ depends only on $\Omega, C_{\varphi}, M_{m}$.

Proposition 3.3. Assume that $m, C_{3}$ satisfy Proposition 3.2 and $\frac{2}{m}<\epsilon<T$. Then there exists $C_{4}=C_{4}\left(\Omega, m, \epsilon, T, C_{f}, C_{1}, C_{2}, C_{3}\right)>0$ such that 


$$
|\nabla u| \leq C_{4} \text { on } \Omega \times(\epsilon, T) .
$$

Proof. We will use the technique of Blocki as in [Blo08]. In this proof only, we denote

$$
\begin{gathered}
g(t)=n \log \left(t-\frac{2}{m}\right), \\
\gamma(u)=A u-B u^{2} \quad \text { where } A=\frac{1}{10 C_{1}}, B=\frac{1}{20 C_{1}^{2}}, \\
\eta=\frac{1}{4(\operatorname{diam} \Omega)^{2}}, \\
\phi=\log |\nabla u|^{2}+\gamma(u)+g(t)+\eta|z|^{2},
\end{gathered}
$$

and we assume that $0 \in \Omega$.

Let $\epsilon<T^{\prime}<T$, we will prove that

$$
\sup _{\Omega \times\left(\frac{2}{m}, T^{\prime}\right)} \phi \leq \tilde{C}_{4}
$$

where $\tilde{C}_{4}$ depends on $\Omega, C_{1}, C_{2}, C_{3}, m, T, C_{f}$.

Notice that the hypotheses and previous bounds on $|u|$ imply that, for $t \in\left(\frac{2}{m}, T^{\prime}\right)$,

$$
\exp \phi(z, t) \leq|\nabla u(z, t)|^{2}\left(t-\frac{2}{m}\right)^{n} \exp \left(\max _{\Omega \times\left(\frac{2}{m}, T^{\prime}\right)} \gamma(u)+\eta \max _{\Omega}|z|\right) \leq C|\nabla u|^{2}
$$

and in a similar way

$$
|\nabla u(z, t)|^{2} \leq C\left(\epsilon-\frac{2}{m}\right)^{-n} \exp \phi(z, t) \leq C_{\epsilon} \exp \phi(z, t), \quad t \in\left(\epsilon, T^{\prime}\right),
$$

so the bound on $\phi$ yields a bound on $|\nabla u(z, t)|$.

Suppose that

$$
\sup _{\Omega \times\left(\frac{2}{m}, T^{\prime}\right)} \phi=\phi\left(z_{0}, t_{0}\right) .
$$

By an orthogonal change of coordinates, we can assume that $\left(u_{\alpha \bar{\beta}}\left(z_{0}, t_{0}\right)\right)$ is diagonal. For convenience, we denote $u_{\alpha \bar{\alpha}}\left(z_{0}, t_{0}\right)=\lambda_{\alpha}$.

We also denote by $\mathcal{L}$ the operator

$$
\mathcal{L}=\frac{\partial}{\partial t}-\sum u^{\alpha \bar{\beta}} \frac{\partial^{2}}{\partial z_{\alpha} \partial \bar{z}_{\beta}} .
$$

If $|\nabla u|^{2}\left(z_{0}, t_{0}\right) \leq C$, by (17), we are done. In particular, if $z_{0} \in \partial \Omega$, we know that $|\nabla u(z, t)|$ is bounded. So we may restrict attention to the case where $|\nabla u|^{2}\left(z_{0}, t_{0}\right)>1$ and $\left(z_{0}, t_{0}\right) \in \Omega \times\left(\frac{2}{m}, T^{\prime}\right]$. Then $\left.\mathcal{L}(\phi)\right|_{\left(z_{0}, t_{0}\right)} \geq 0$.

We compute

$$
\begin{aligned}
\mathcal{L}(\phi) & =\mathcal{L}\left(\log |\nabla u|^{2}\right)+\gamma^{\prime}(u) \cdot \dot{u}+g^{\prime}(t)-\gamma^{\prime}(u) \sum u^{\alpha \bar{\beta}} u_{\alpha \bar{\beta}} \\
& -\gamma^{\prime \prime}(u) \sum u^{\alpha \bar{\beta}} u_{\alpha} u_{\bar{\beta}}-\eta \sum u^{\alpha \bar{\alpha}} \\
& =\mathcal{L}\left(\log |\nabla u|^{2}\right)+\gamma^{\prime}(u) \cdot(\dot{u}-n)+g^{\prime}(t) \\
& -\gamma^{\prime \prime}(u) \sum u^{\alpha \bar{\beta}} u_{\alpha} u_{\bar{\beta}}-\eta \sum u^{\alpha \bar{\alpha}} .
\end{aligned}
$$


When $|\nabla u| \neq 0$, we have

$$
\begin{aligned}
\left(\log |\nabla u|^{2}\right)_{\alpha \bar{\beta}} & =\frac{|\nabla u|_{\alpha \bar{\beta}}^{2}}{|\nabla u|^{2}}-\frac{|\nabla u|_{\alpha}^{2}|\nabla u|_{\bar{\beta}}^{2}}{|\nabla u|^{4}} \\
& =\frac{\left\langle\nabla u_{\alpha \bar{\beta}}, \nabla u\right\rangle}{|\nabla u|^{2}}+\frac{\left\langle\nabla u, \nabla u_{\beta \bar{\alpha}}\right\rangle}{|\nabla u|^{2}}+\frac{\left\langle\nabla u_{\alpha}, \nabla u_{\beta}\right\rangle}{|\nabla u|^{2}} \\
& +\frac{\left\langle\nabla u_{\bar{\beta}}, \nabla u_{\bar{\alpha}}\right\rangle}{|\nabla u|^{2}}-\frac{|\nabla u|_{\alpha}^{2}|\nabla u|_{\bar{\beta}}^{2}}{|\nabla u|^{4}} . \\
\mathcal{L}\left(\log |\nabla u|^{2}\right) & =\frac{\langle\nabla \dot{u}, \nabla u\rangle-\sum\left\langle u^{\alpha \bar{\beta}} \nabla u_{\alpha \bar{\beta}}, \nabla u\right\rangle}{|\nabla u|^{2}}+\frac{\langle\nabla u, \nabla \dot{u}\rangle-\sum\left\langle\nabla u, u^{\beta \bar{\alpha}} \nabla u_{\beta \bar{\alpha}}\right\rangle}{|\nabla u|^{2}} \\
& -\sum u^{\alpha \bar{\beta}} \frac{\left\langle\nabla u_{\alpha}, \nabla u_{\beta}\right\rangle+\left\langle\nabla u_{\bar{\beta}}, \nabla u_{\bar{\alpha}}\right\rangle}{|\nabla u|^{2}}+\sum u^{\alpha \bar{\beta}} \frac{\left(|\nabla u|^{2}\right)_{\alpha}\left(|\nabla u|^{2}\right)_{\bar{\beta}}}{|\nabla u|^{4}} .
\end{aligned}
$$

We have, by (7),

$$
\begin{aligned}
\left.\mathcal{L}\left(\log |\nabla u|^{2}\right)\right|_{\left(z_{0}, t_{0}\right)} & =2 \operatorname{Re}\left(\frac{\langle\nabla u, \nabla f\rangle}{|\nabla u|^{2}}\right)+2 f_{u}(t, z, u)|\nabla u|^{2}-\sum \frac{\left|\nabla u_{k}\right|^{2}+\left|\nabla u_{\bar{k}}\right|^{2}}{\lambda_{k}|\nabla u|^{2}} \\
& +\sum \frac{\left(|\nabla u|^{2}\right)_{k}\left(|\nabla u|^{2}\right)_{\bar{k}}}{\lambda_{k}|\nabla u|^{4}} \\
& \leq \frac{2|\nabla f|}{|\nabla u|}+\sum \frac{\left(|\nabla u|^{2}\right)_{k}\left(|\nabla u|^{2}\right)_{\bar{k}}}{\lambda_{k}|\nabla u|^{4}} .
\end{aligned}
$$

Hence, there exists $C_{4}^{\prime}=C_{4}^{\prime}\left(m, C_{1}, C_{2}, C_{f}\right)$ such that

$$
\left.\mathcal{L}(\phi)\right|_{\left(z_{0}, t_{0}\right)} \leq C_{4}^{\prime}+g^{\prime}(t)-\gamma^{\prime \prime}(u) \sum \frac{\left|u_{k}\right|^{2}}{\lambda_{k}}-\eta \sum \frac{1}{\lambda_{k}}+\sum \frac{\left(|\nabla u|^{2}\right)_{k}\left(|\nabla u|^{2}\right)_{\bar{k}}}{\lambda_{k}|\nabla u|^{4}}
$$

By the condition $\frac{\partial \phi}{\partial z_{k}}\left(z_{0}, t_{0}\right)=0$, we have

$$
\frac{\left(|\nabla u|^{2}\right)_{k}\left(|\nabla u|^{2}\right)_{\bar{k}}}{|\nabla u|^{4}}=\left|\gamma^{\prime}(u) u_{k}+\eta \bar{z}_{k}\right|^{2} \leq 2\left(\gamma^{\prime}(u)\right)^{2}\left|u_{k}\right|^{2}+2 \eta^{2}\left|z_{k}\right|^{2} \leq 2\left(\gamma^{\prime}(u)\right)^{2}\left|u_{k}\right|^{2}+\frac{\eta}{2},
$$

where $(z, t)=\left(z_{0}, t_{0}\right)$.

Then

$$
\begin{aligned}
0 \leq\left.\mathcal{L}(\phi)\right|_{\left(z_{0}, t_{0}\right)} & \leq C_{4}^{\prime}+g^{\prime}(t)+\left(2\left(\gamma^{\prime}(u)\right)^{2}-\gamma^{\prime \prime}(u)\right) \sum \frac{\left|u_{k}\right|^{2}}{\lambda_{k}}-\frac{\eta}{2} \sum \frac{1}{\lambda_{k}} \\
& \leq C_{4}^{\prime}+g^{\prime}(t)-a\left(\sum \frac{\left|u_{k}\right|^{2}}{\lambda_{k}}+\sum \frac{1}{\lambda_{k}}\right)
\end{aligned}
$$

where $a:=\min \left\{2 B-\left(A+B C_{1}\right), \frac{\eta}{2}\right\}$. Hence, at $\left(z_{0}, t_{0}\right)$

$$
\sum \frac{\left|u_{k}\right|^{2}}{\lambda_{k}}+\sum \frac{1}{\lambda_{k}} \leq \frac{1}{a}\left(C_{4}^{\prime}+g^{\prime}(t)\right)
$$

Moreover, by Proposition 3.1 and by (16), there exists $C_{4}^{\prime \prime}=C_{4}^{\prime \prime}\left(m, C_{1}, C_{2}\right)$ such that

$$
\lambda_{1} \lambda_{2} \ldots \lambda_{n}=\operatorname{det}\left(u_{\alpha \bar{\beta}}\right)=e^{\dot{u}-f(t, z, u)} \leq C_{4}^{\prime \prime} .
$$


By (18) and (19), there exists $C_{4}^{\prime \prime \prime}=C_{4}^{\prime \prime \prime}\left(a, C_{4}^{\prime}, C_{4}^{\prime \prime}\right)$ such that

$$
\begin{gathered}
\lambda_{k}=\prod \lambda_{j} \prod_{l \neq k} \frac{1}{\lambda_{l}} \leq\left(C_{4}^{\prime \prime \prime}+g^{\prime}\left(t_{0}\right)\right)^{n-1} \quad \text { for } \quad k=1, \ldots, n . \\
|\nabla u|^{2}=\sum\left|u_{k}\right|^{2} \leq\left(\left(C_{4}^{\prime \prime \prime}+g^{\prime}\left(t_{0}\right)\right)^{n} \text { for } \quad(z, t)=\left(z_{0}, t_{0}\right) .\right.
\end{gathered}
$$

Then

$$
\begin{aligned}
\phi\left(z_{0}, t_{0}\right) & \leq n \log \left(C_{4}^{\prime \prime \prime}+g^{\prime}\left(t_{0}\right)\right)+g\left(t_{0}\right)+\gamma\left(u\left(z_{0}, t_{0}\right)\right)+\eta\left|z_{0}\right|^{2} \\
& \leq n \log \left(C_{4}^{\prime \prime \prime}\left(t_{0}-\frac{2}{m}\right)+n\right)+\gamma\left(u\left(z_{0}, t_{0}\right)\right)+\eta\left|z_{0}\right|^{2} \\
& \leq \tilde{C}_{4}
\end{aligned}
$$

For $z \in \Omega, \frac{2}{m}<\epsilon<t<T^{\prime}$, we have

$$
\log |\nabla u|^{2} \leq \tilde{C}_{4}-\gamma(u)-\eta|z|^{2}-g(t) \leq 2 \log C_{4},
$$

where $C_{4}>0$ depends on $\Omega, m, \epsilon, T, C_{f}, C_{1}, C_{2}, C_{3}$.

\section{Higher order estimates}

In this section, we prove that the second derivatives of $u$ are bounded on $\partial \Omega \times(\epsilon, T)$. Then we use the maximum principle to show that the Laplacian of $u$ is bounded on $\Omega \times(\epsilon, T)$. For convenience, we denote $\underline{u}:=\underline{u}_{m}, M:=M_{m}$, where $\frac{1}{2 m}<\epsilon \leq \frac{1}{2 m-1}$ and $u_{m}, M_{m}$ are defined as in 2.4 .

\subsection{Localisation technique.}

In order to show that the second derivatives of $u$ are bounded on $\partial \Omega \times(\epsilon, T)$, we use a barrier function. The key to the construction is the following:

Lemma 4.1. We set

$$
v=(u-\underline{u})+a(h-\underline{u})-N d^{2},
$$

where $d$ is the distance from $\partial \Omega, h$ is defined as in the proof of Proposition 3.2 and $a, N$ are positive constants to be determined. Let $\epsilon \in(0, T)$. Then there exist $a, N, \delta>0$ depending only on $\Omega, \epsilon, T, C_{u}, C_{\varphi}, C_{f}$ such that

$$
\begin{gathered}
L(v) \geq \frac{1}{4}\left(1+\sum u^{\alpha \bar{\alpha}}\right) \quad \text { on } \quad U_{\delta} \times(\epsilon, T), \\
v \geq 0 \quad \text { on } \quad U_{\delta} \times(\epsilon, T),
\end{gathered}
$$

where $U_{\delta}=\{z \in \Omega: d(z) \leq \delta\}$.

Proof. The elliptic version of this lemma was proved by [Gua98] (page 5-7). The same arguments can be applied for the parabolic case. For the reader's convenience, we recall the arguments here.

We have

$$
L(v)=\dot{v}-n+\sum u^{\alpha \bar{\beta}} \underline{u}_{\alpha \bar{\beta}}-a \sum u^{\alpha \bar{\beta}}\left(h_{\alpha \bar{\beta}}-\underline{u}_{\alpha \bar{\beta}}\right)+2 N \sum u^{\alpha \bar{\beta}}\left(d d_{\alpha \bar{\beta}}+d_{\alpha} d_{\bar{\beta}}\right)-f_{u}(t, z, u) v .
$$


Fix $\tilde{\delta}>0$ satisfying $d \in C^{\infty}\left(U_{\tilde{\delta}}\right)$. Assume that $0<a<1$ and $0<\delta<\tilde{\delta}$ and $0<N<\frac{1}{\delta}$. Then there exists $C_{5}>0$ depending on $\Omega, \tilde{\delta}, \epsilon, T, C_{\varphi}, C_{f}, M, C_{1}, C_{2}$ such that

$$
\begin{gathered}
\dot{v}-n-f_{u}(t, z, u) v \geq-C_{5}, \\
-a \sum u^{\alpha \bar{\beta}}\left(h_{\alpha \bar{\beta}}-\underline{u}_{\alpha \bar{\beta}}\right) \geq-C_{5} a \sum u^{\alpha \bar{\alpha}}, \\
2 N d \sum u^{\alpha \bar{\beta}} d_{\alpha \bar{\beta}} \geq-C_{5} N \delta \sum u^{\alpha \bar{\alpha}},
\end{gathered}
$$

where $(z, t) \in U_{\delta} \times(\epsilon, T)$.

Then

$$
L(v) \geq \sum u^{\alpha \bar{\beta}} \underline{u}_{\alpha \bar{\beta}}-C_{5}-C_{5}(a+N \delta) \sum u^{\alpha \bar{\alpha}}+2 N \sum u^{\alpha \bar{\beta}} d_{\alpha} d_{\bar{\beta}},
$$

where $(z, t) \in U_{\delta} \times(\epsilon, T)$.

When $a+N \delta \leq \frac{1}{4 C_{5}}$, we obtain

$$
L(v) \geq \frac{3}{4} \sum u^{\alpha \bar{\alpha}}-C_{5}+2 N \sum u^{\alpha \bar{\beta}} d_{\alpha} d_{\bar{\beta}}
$$

where $(z, t) \in U_{\delta} \times(\epsilon, T)$.

Let $\lambda_{1} \leq \lambda_{2} \leq \ldots \leq \lambda_{n}$ be the eigenvalues of $\left\{u_{\alpha \bar{\beta}}\right\}$. We have

$$
\sum u^{\alpha \bar{\beta}} d_{\alpha} d_{\bar{\beta}} \geq \lambda_{n}^{-1} \sum d_{\alpha} d_{\bar{\alpha}} \geq \frac{\lambda_{n}^{-1}}{2} \quad \text { on } \quad U_{\delta} \times(\epsilon, T) .
$$

By the inequality for arithmetic and geometric means

$$
\frac{1}{4} \sum u^{\alpha \bar{\alpha}}+N \lambda_{n}^{-1} \geq n\left(\frac{1}{4}\right)^{(n-1) / n} N^{1 / n}\left(\lambda_{1} \ldots \lambda_{n}\right)^{-1 / n} \geq C_{6} N^{1 / n},
$$

where $C_{6}>0$ depends on $\epsilon, T, C_{f}, C_{1}, C_{2}$.

When $N>\left(\frac{C_{5}+1}{C_{6}}\right)^{n}$, we have

$$
L(v) \geq \frac{1}{2}\left(2+\sum u^{\alpha \bar{\alpha}}\right) .
$$

Next, since $\Delta \underline{u} \geq n$, there exists $C_{7}>0$ depending only on $\Omega$ such that

$$
(h-\underline{u}) \geq C_{7} d \quad \text { on } \quad \Omega \times(\epsilon, T) .
$$

Fix $0<a, \delta<1, N>0$ so that

$$
\begin{aligned}
& \text { - } N>\left(\frac{C_{5}+1}{C_{6}}\right)^{n} ; \\
& \text { - } a \leq \frac{1}{8 C_{5}} \\
& \text { - } 0<\delta<\tilde{\delta} \\
& \text { - } \min \left\{a C_{7}, a\right\} \geq N \delta .
\end{aligned}
$$

We obtain

$$
\begin{gathered}
L(v) \geq \frac{1}{4}\left(1+\sum u^{\alpha \bar{\alpha}}\right) \quad \text { on } \quad U_{\delta} \times(\epsilon, T), \\
v \geq 0 \quad \text { on } \quad U_{\delta} \times(\epsilon, T) .
\end{gathered}
$$




\section{2. $\mathcal{C}^{2}$-a priori estimates on the boundary.}

Lemma 4.2. Let $\epsilon \in(0, T)$. Then there exists $c_{\epsilon}>0$ depending only on $\Omega, \epsilon, T, C_{u}, C_{\varphi}, C_{f}$ such that

$$
\left.\left(d d^{c} u\right)\right|_{T_{\partial \Omega}^{h}} \geq\left. c_{\epsilon}\left(d d^{c}|z|^{2}\right)\right|_{T_{\partial \Omega}^{h}},
$$

where $T_{\partial \Omega}^{h}$ is the holomorphic tangent bundle of $\partial \Omega$.

We refer the reader to [CKNS85, pp. 221-223] or [Bou11, p. 268-271] for related results in the elliptic case.

Proof. Fix $p \in \partial \Omega$. By an affine change of coordinates, we can assume that $p=0$ and there exists a neighbourhood $U$ of $p$ such that

$$
\Omega \cap U=\left\{z \in U: x_{n}>\operatorname{Re}\left(\sum_{1 \leq j \leq k \leq n} a_{j \bar{k}} z_{j} \bar{z}_{k}+\sum_{1 \leq j \leq k \leq n} a_{j k} z_{j} z_{k}\right)+O\left(|z|^{3}\right)\right\},
$$

where $a_{j \bar{k}}, a_{j k} \in \mathbb{C}$ with $a_{1 \overline{1}}>0$.

By a holomorphic change of coordinates, we can assume that

$$
\Omega \cap U=\left\{z \in U: x_{n}>\operatorname{Re}\left(\sum_{1 \leq j \leq k \leq n} a_{j \bar{k}} z_{j} \bar{z}_{k}\right)+O\left(|z|^{3}\right)\right\}
$$

where $a_{j \bar{k}}$ with $a_{1 \overline{1}}>0$.

We need to show that

$$
u_{1 \overline{1}}(p, t) \geq C_{\epsilon},
$$

where $t \in(\epsilon, T)$ and $C_{\epsilon}>0$ depends on $\Omega, \epsilon, T, C_{u}, C_{\varphi}, C_{f}$.

Step 1: Choice of a Kähler potential.

We construct a function $\tau \in C^{\infty}\left(\Omega_{r} \times(\epsilon, T)\right)$ depending on $\underline{u}, \epsilon, T, \Omega$ so that $d d^{c} \tau=d d^{c} \underline{u}$ and $\tau(p, t)=0$ and

$$
\left.\tau\right|_{\left(\partial \Omega \cap B_{r}\right) \times(\epsilon, T)}=\operatorname{Re}\left(\sum_{j=2}^{n} c_{j} z_{1} \bar{z}_{j}\right)+O\left(\left|z_{2}\right|^{2}+\ldots+\left|z_{n}\right|^{2}\right),
$$

where $r>0, B_{r}=B_{r}(p), \Omega_{r}=\Omega \cap B_{r}$ and $c_{j} \in C^{\infty}([\epsilon, T), \mathbb{C})$.

Indeed, by Taylor's formula,

$$
\begin{aligned}
\underline{u}(z, t)-\underline{u}(p, t) & =\operatorname{Re}\left(\sum_{j=1}^{n} b_{j} z_{j}\right)+\operatorname{Re}\left(\sum_{j=2}^{n} b_{1 \bar{j}} z_{1} \bar{z}_{j}\right)+b_{1 \overline{1}}\left|z_{1}\right|^{2}+\operatorname{Re}\left(\sum_{j=1}^{n} b_{1 j} z_{1} z_{j}\right) \\
& +O\left(\left|z_{2}\right|^{2}+\ldots+\left|z_{n}\right|^{2}\right)+O\left(|z|^{3}\right),
\end{aligned}
$$

where $b_{j}, b_{1 j}, b_{1 \bar{j}} \in \mathbb{C}^{\infty}([\epsilon, T), \mathbb{C}), b_{1 \overline{1}}=\underline{u}_{1 \overline{1}}(p, t)>0$.

Furthermore, near $p$ on $\partial \Omega$, we have by (20)

$$
x_{n}=\operatorname{Re}\left(\sum_{j=2}^{n} a_{1 \bar{j}} z_{1} \bar{z}_{j}\right)+a_{1 \overline{1}}\left|z_{1}\right|^{2}+O\left(\left|z_{2}\right|^{2}+\ldots+\left|z_{n}\right|^{2}\right)+O\left(|z|^{3}\right),
$$

where $a_{1 \bar{j}} \in \mathbb{C}$ with $a_{1 \overline{1}}>0$.

Define

$$
\tau(z, t)=\underline{u}(z, t)-\underline{u}(p, t)-\operatorname{Re}\left(\sum_{j=1}^{n} b_{j} z_{j}\right)-\frac{b_{1 \overline{1}}}{a_{1 \overline{1}}} x_{n}-\operatorname{Re}\left(\sum_{j=1}^{n} b_{1 j} z_{1} z_{j}\right)
$$


then $d d^{c} \tau=d d^{c} \underline{u}$ and $\tau(p, t)=0$ and

$$
\left.\tau\right|_{\left(\partial \Omega \cap B_{r}\right) \times(\epsilon, T)}=\operatorname{Re}\left(\sum_{j=2}^{n} c_{j} z_{1} \bar{z}_{j}\right)+O\left(\left|z_{2}\right|^{2}+\ldots+\left|z_{n}\right|^{2}\right)+\{\text { terms of order } \geq 3\} .
$$

Moreover, for $z \in \partial \Omega$, we have

- For $j=2, \ldots, n$

$$
\left|z_{j}\right|^{2}\left|z_{1}\right|=O\left(\left|z_{2}\right|^{2}+\ldots+\left|z_{n}\right|^{2}\right)
$$

- By (21)

$$
\begin{aligned}
\left|z_{1}\right|^{4} & =O\left(x_{n}^{2}\right)+O\left(\sum_{j=2}^{n}\left|z_{1}\right|^{2}\left|z_{j}\right|^{2}\right)+O\left(|z|^{6}\right)+O\left(\left(\sum_{j=2}^{n}\left|z_{j}\right|^{2}\right)^{2}\right) \\
& =O\left(\left|z_{2}\right|^{2}+\ldots+\left|z_{n}\right|^{2}\right)+O\left(|z|^{6}\right)
\end{aligned}
$$

then

$$
|z|^{4}=O\left(\left|z_{2}\right|^{2}+\ldots+\left|z_{n}\right|^{2}\right)
$$

- For $j=2, \ldots, n$

$$
\left|z_{1}\right|^{2}\left|z_{j}\right|=O\left(\left|z_{1}\right|^{4}\right)+O\left(\left|z_{j}\right|^{2}\right)=O\left(\left|z_{2}\right|^{2}+\ldots+\left|z_{n}\right|^{2}\right) .
$$

Hence

$$
\begin{aligned}
\left.\tau\right|_{\left(\partial \Omega \cap B_{r}\right) \times(\epsilon, T)} & =\operatorname{Re}\left(\sum_{j=2}^{n} c_{j} z_{1} \bar{z}_{j}\right)+\sum \tilde{a}_{j} x_{1}^{j} y_{1}^{3-j}+O\left(\left|z_{2}\right|^{2}+\ldots+\left|z_{n}\right|^{2}\right) \\
& =\operatorname{Re}\left(\sum_{j=2}^{n} c_{j} z_{1} \bar{z}_{j}\right)+\operatorname{Re}\left(a_{1} z_{1}^{3}\right)+\operatorname{Re}\left(a_{2} z_{1}\left|z_{1}\right|^{2}\right)+O\left(\left|z_{2}\right|^{2}+\ldots+\left|z_{n}\right|^{2}\right),
\end{aligned}
$$

where $a_{1}, a_{2} \in C^{\infty}([\epsilon, T), \mathbb{C})$.

Next, by (21), (22), (24), for $z \in \partial \Omega$, we have

$$
\begin{aligned}
\operatorname{Re}\left(a_{2} z_{1}\left|z_{1}\right|^{2}\right) & =\operatorname{Re}\left(\frac{a_{2}}{a_{1 \overline{1}}} z_{1} x_{n}\right)+O\left(\left|z_{2}\right|^{2}+\ldots+\left|z_{n}\right|^{2}\right) \\
& =\operatorname{Re}\left(c_{0} z_{1} \bar{z}_{n}\right)+\operatorname{Re}\left(c_{0} z_{1} z_{n}\right)+O\left(\left|z_{2}\right|^{2}+\ldots+\left|z_{n}\right|^{2}\right) .
\end{aligned}
$$

Replacing the term $c_{n}$ by $c_{n}-c_{0}$, we obtain

$$
\left.\tau\right|_{\left(\partial \Omega \cap B_{r}\right) \times(\epsilon, T)}=\operatorname{Re}\left(\sum_{j=2}^{n} c_{j} z_{1} \bar{z}_{j}\right)+\operatorname{Re}\left(a_{1} z_{1}^{3}\right)+\operatorname{Re}\left(c_{0} z_{1} z_{n}\right)+O\left(\left|z_{2}\right|^{2}+\ldots+\left|z_{n}\right|^{2}\right) .
$$

Replacing $\tau$ by $\tau+\operatorname{Re}\left(a_{1} z_{1}^{3}\right)+\operatorname{Re}\left(c_{0} z_{1} z_{n}\right)$, we obtain

$$
\left.\tau\right|_{\left(\partial \Omega \cap B_{r}\right) \times(\epsilon, T)}=\operatorname{Re}\left(\sum_{j=2}^{n} c_{j} z_{1} \bar{z}_{j}\right)+O\left(\left|z_{2}\right|^{2}+\ldots+\left|z_{n}\right|^{2}\right) .
$$

Therefore,

(25) $\left.\tau\right|_{\left(\partial \Omega \cap B_{r}\right) \times(\epsilon, T)} \leq \operatorname{Re}\left(\sum_{j=2}^{n} c_{j} z_{1} \bar{z}_{j}\right)+a_{3}\left(\left|z_{2}\right|^{2}+\ldots+\left|z_{n}\right|^{2}\right), \quad \sup \sum_{j=2}^{n}\left|c_{j}\right| \leq a_{4}$,

where $a_{3}, a_{4}>0$ depend on $\Omega, \epsilon, T, M, C_{\varphi}$.

The conditions $d d^{c} \tau=d d^{c} \underline{u}$ and $\tau(p, t)=0$ are still satisfied. 
Step 2: Choice of a barrier function.

Recall that $\Omega_{r}=\Omega \cap B_{r}$. We construct a function

$$
b(z, t)=-\epsilon_{1} x_{n}+\epsilon_{2}|z|^{2}+\frac{1}{2 \mu} \sum_{j=2}^{n}\left|c_{j} z_{1}+\mu z_{j}\right|^{2}
$$

such that $b \geq \tau+u-\underline{u}$ on $\Omega_{r} \times(\epsilon, T)$, where $r>0$ depends only on $\Omega$ and $\epsilon_{1}, \epsilon_{2}, \mu>0$ depend on $\Omega, \epsilon, T, M, C_{\varphi}, C_{f}$.

Note that

$$
\left|z_{1}\right|^{2} \leq \frac{1}{a_{1 \overline{1}}}\left(x_{n}-\operatorname{Re}\left(\sum_{j=2}^{n} a_{1 \bar{j}} z_{1} \bar{z}_{j}\right)\right)+O\left(\left|z_{2}\right|^{2}+\ldots+\left|z_{n}\right|^{2}\right)+O\left(|z|^{3}\right) \text { on } \Omega .
$$

Since for $r_{0}$ small enough and $z \in \Omega_{r_{0}}$, we have $z \rightarrow 0$ as $\left|z_{2}\right|^{2}+\ldots+\left|z_{n}\right|^{2} \rightarrow 0$, if we fix $r>0$ small enough, then there exists $r_{1}>0$ such that

$$
\left|z_{2}\right|^{2}+\ldots+\left|z_{n}\right|^{2} \geq r_{1} \quad \text { for } \quad z \in \partial B_{r} \cap \Omega \text {. }
$$

Assume that $0<\epsilon_{1}, \epsilon_{2}<1$. Then there exists $\mu_{1}>0$ depending on $\Omega, M, C_{\varphi}, C_{1}, a_{3}, a_{4}, r_{1}$ such that the function $b$ in (26) verifies

$$
\begin{aligned}
\left.b\right|_{\left(\partial B_{r}(p) \cap \Omega\right) \times[\epsilon, T)} & \geq \frac{\mu r_{1}}{2}+\operatorname{Re}\left(\sum_{j=2}^{n} c_{j} z_{1} \bar{z}_{j}\right)-\epsilon_{1} x_{n}+\epsilon_{2}|z|^{2} \\
& \geq \frac{\mu_{1} r_{1}}{2}+\operatorname{Re}\left(\sum_{j=2}^{n} c_{j} z_{1} \bar{z}_{j}\right)-\epsilon_{1} x_{n}+\epsilon_{2}|z|^{2} \\
& \geq\left.(\tau+u-\underline{u})\right|_{\left(\partial B_{r}(p) \cap \Omega\right) \times[\epsilon, T)}
\end{aligned}
$$

when $\mu \geq \mu_{1}$.

There exists $r_{2}>0$ such that, when $z \in \partial \Omega$,

$$
x_{n}=\operatorname{Re}\left(\sum_{j=1}^{n} a_{1 \bar{j}} z_{1} \bar{z}_{j}\right)+O\left(\left|z_{2}\right|^{2}+\ldots+\left|z_{n}\right|^{2}\right)+O\left(|z|^{3}\right) \leq r_{2}|z|^{2} .
$$

Assume that $0<r_{2} \epsilon_{1}<\epsilon_{2}$. For $\mu \geq 2 a_{3}$, by (25), we have

$$
\begin{aligned}
\left.b\right|_{\left(\partial \Omega \cap B_{r}(p)\right) \times[\epsilon, T)} & \geq \frac{1}{2 \mu} \sum_{j=2}^{n}\left|c_{j} z_{1}+\mu z_{j}\right|^{2} \\
& \geq \operatorname{Re}\left(\sum_{j=2}^{n} c_{j} z_{1} \bar{z}_{j}\right)+\frac{\mu}{2}\left(\left|z_{2}\right|^{2}+\ldots+\left|z_{n}\right|^{2}\right) \\
& \geq\left.\tau\right|_{\left(\partial \Omega \cap B_{r}(p)\right) \times[\epsilon, T)} \\
& \geq\left.(\tau+u-\underline{u})\right|_{\left(\partial \Omega \cap B_{r}(p)\right) \times[\epsilon, T)} .
\end{aligned}
$$

Fix $\mu \geq \max \left(\mu_{1}, 2 a_{3}\right)$, we get

$$
\left.b\right|_{\partial_{P}\left(\Omega_{r} \times[\epsilon, T)\right)} \geq\left.(\tau+u-\underline{u})\right|_{\partial \Omega_{r} \times[\epsilon, T)} .
$$

Next, by Proposition 3.1, there exists $r_{3}>0$ such that

$$
\left(d d^{c}(\tau-u-\underline{u})\right)^{n}=\left(d d^{c} u\right)^{n}=e^{\dot{u}-f(t, z, u)} \geq r_{3} \quad \text { on } \quad \Omega_{r} \times[\epsilon, T) .
$$


On the other hand

$$
\left(d d^{c}\left(\sum_{j=2}^{n}\left|c_{j} z_{1}+\mu z_{j}\right|^{2}\right)\right)^{n}=0,
$$

so $\left(d d^{c} b\right)^{n}=O\left(\epsilon_{2}\right)$ on $\Omega_{r} \times[\epsilon, T)$.

Hence, there exists $\epsilon_{2}>0$ depending on $\mu, \Omega, a_{4}, r_{3}$ such that

$$
\left(d d^{c} b\right)^{n} \leq\left(d d^{c}(\tau+u-\underline{u})\right)^{n} \text { on } \Omega_{r} \times[\epsilon, T) .
$$

When $\left.b\right|_{\partial \Omega_{r} \times[\epsilon, T)} \geq\left.(\tau+u-\underline{u})\right|_{\partial \Omega_{r} \times[\epsilon, T)}$ and $\left(d d^{c} b\right)^{n} \leq\left(d d^{c}(\tau+u-\underline{u})\right)^{n}$ on $\Omega_{r} \times[\epsilon, T)$, it follows from the comparison theorem (for the bounded plurisubharmonic functions) that

$$
b \geq(\tau+u-\underline{u}) \quad \text { on } \quad \Omega_{r} \times[\epsilon, T) .
$$

Step 3: Conclusion.

We have, since $b(p, t)=\tau(p, t)+u(p, t)-\underline{u}(p, t)=0$,

$$
-\epsilon_{1}=b_{x_{n}}(p, t) \geq \tau_{x_{n}}(p, t)+(u-\underline{u})_{x_{n}}(p, t) .
$$

Then, since $\left.(u-\underline{u})\right|_{\partial \Omega \times(\epsilon, T)} \equiv 0$,

$$
(u-\underline{u})_{1 \overline{1}}(p, t)=-(u-\underline{u})_{x_{n}}(p, t) \rho_{1 \overline{1}}(p),
$$

and by the explicit choice of $\tau,-\tau_{x_{n}}(p, t) \rho_{1 \overline{1}}(p)=\tau_{1 \overline{1}}(p, t)$, so

$$
u_{1 \overline{1}}(p, t)=\left(\tau_{1 \overline{1}}+u_{1 \overline{1}}-\underline{u}_{1 \overline{1}}\right)(p, t)=-\left(\tau_{x_{n}}(p, t)+(u-\underline{u})_{x_{n}}(p, t)\right) \rho_{1 \overline{1}}(p) \geq \epsilon_{1} \rho_{1 \overline{1}}(p) .
$$

Proposition 4.3. There exists $D_{1}=D_{1}\left(\Omega, \epsilon, T, C_{u}, C_{\varphi}, C_{f}\right)$ such that

$$
\left|D^{2} u\right| \leq D_{1} \quad \text { on } \quad \partial \Omega \times(\epsilon, T) .
$$

Proof. Fix $p \in \partial \Omega$. We can choose complex coordinates $\left(z_{j}\right)_{1 \leq j \leq n}$ so that $p=0$ and the positive $x_{n}$ axis is the interior normal direction of $\partial \Omega$ at $p$. We set for convenience

$$
s_{1}=y_{1}, s_{2}=x_{1}, \ldots, s_{2 n-1}=y_{n}, s_{2 n}=x_{n}, s^{\prime}=\left(s_{1}, \ldots, s_{2 n-1}\right) .
$$

We also assume that near $p, \partial \Omega$ is represented as a graph

$$
x_{n}=P\left(s^{\prime}\right)=\sum_{j, k<2 n} P_{j k} s_{j} s_{k}+O\left(\left|s^{\prime}\right|^{3}\right) .
$$

Step 1: Bounding the tangent-tangent derivatives.

Since $(u-\underline{u})\left(s^{\prime}, P\left(s^{\prime}\right), t\right)=0$, we have for $j, k<2 n, 0<t<T$ :

$$
(u-\underline{u})_{s_{j} s_{k}}(p, t)=-(u-\underline{u})_{x_{n}}(p, t) P_{j k} .
$$

By Proposition 3.2, we obtain

$$
\left|u_{s_{j} s_{k}}(p, t)\right| \leq D_{1}^{\prime},
$$

where $D_{1}^{\prime}>0$ depends only on $\Omega, C_{\varphi}, M$.

Step 2: Bounding the normal-tangent derivatives. 
Define

$$
T_{j}=\frac{\partial}{\partial s_{j}}+P_{s_{j}} \frac{\partial}{\partial x_{n}} .
$$

Again, denote $\Omega_{\delta}=B_{\delta}(p) \cap \Omega$. With $v$ as in Lemma 4.2, we construct the functions

$$
\psi_{ \pm}=A v+B|z|^{2}-\left(t-\frac{\epsilon}{2}\right)\left(u_{y_{n}}-\underline{u}_{y_{n}}\right)^{2} \pm\left(t-\frac{\epsilon}{2}\right) T_{j}(u-\underline{u}),
$$

such that

$$
\begin{gathered}
L\left(\psi_{ \pm}\right) \geq 0 \quad \text { on } \quad \Omega_{\delta} \times\left(\frac{\epsilon}{2}, T\right), \\
\psi_{ \pm} \geq 0 \quad \text { on } \quad \Omega_{\delta} \times\left(\frac{\epsilon}{2}, T\right),
\end{gathered}
$$

where $A, B>0$ depend on $\Omega, C_{\varphi}, C_{f}, \epsilon, T, M$.

We compute

$$
\begin{aligned}
L\left(-\left(u_{y_{n}}-\underline{u}_{y_{n}}\right)^{2}\right) & =-2\left(u_{y_{n}}-\underline{u}_{y_{n}}\right) L\left(u_{y_{n}}-\underline{u}_{y_{n}}\right)-f_{u}(t, z, u)\left(u_{y_{n}}-\underline{u}_{y_{n}}\right)^{2} \\
& +2 \sum u^{\alpha \bar{\beta}}\left(u_{y_{n}}-\underline{u}_{y_{n}}\right)_{\alpha}\left(u_{y_{n}}-\underline{u}_{y_{n}}\right) \bar{\beta}
\end{aligned}
$$

and

$$
\begin{aligned}
L\left( \pm T_{j}(u-\underline{u})\right) & = \pm L\left(u_{s_{j}}-\underline{u}_{s_{j}}\right) \pm P_{s_{j}} L\left(u_{x_{n}}-\underline{u}_{x_{n}}\right) \\
& \mp\left(u_{x_{n}}-\underline{u}_{x_{n}}\right) \sum u^{\alpha \bar{\beta}}\left(P_{s_{j}}\right)_{\alpha \bar{\beta}} \\
& \mp \sum u^{\alpha \bar{\beta}}\left(\left(u_{x_{n}}-\underline{u}_{x_{n}}\right)_{\alpha}\left(P_{s_{j}}\right)_{\bar{\beta}}+\left(u_{x_{n}}-\underline{u}_{x_{n}}\right)_{\bar{\beta}}\left(P_{s_{j}}\right)_{\alpha}\right) .
\end{aligned}
$$

By equation (7), for $k=1,2, \ldots, 2 n$

$$
L\left(u_{s_{k}}-\underline{u}_{s_{k}}\right)=f_{s_{k}}(t, z, u)-\underline{\dot{u}}_{s_{k}}+\sum u^{\alpha \bar{\beta}}\left(\underline{u}_{s_{k}}\right)_{\alpha \bar{\beta}}+\underline{u}_{s_{k}} f_{u}(t, z, u) .
$$

Hence

$$
\begin{aligned}
& L\left(-\left(u_{y_{n}}-\underline{u}_{y_{n}}\right)^{2} \pm T_{j}(u-\underline{u})\right) \\
& \geq-C_{8}\left(1+\sum u^{\alpha \bar{\alpha}}\right)+2 \sum u^{\alpha \bar{\beta}}\left(u_{y_{n}}-\underline{u}_{y_{n}}\right)_{\alpha}\left(u_{y_{n}}-\underline{u}_{y_{n}}\right)_{\bar{\beta}} \\
& \mp \sum u^{\alpha \bar{\beta}}\left(\left(u_{x_{n}}-\underline{u}_{x_{n}}\right)_{\alpha}\left(P_{s_{j}}\right)_{\bar{\beta}}+\left(u_{x_{n}}-\underline{u}_{x_{n}}\right)_{\bar{\beta}}\left(P_{s_{j}}\right)_{\alpha}\right),
\end{aligned}
$$

where $C_{8}>0$ depend on $\epsilon, C_{1}, C_{2}, C_{3}, M, C_{\varphi}, C_{f}, \rho, P$.

On the other hand

$$
\begin{aligned}
& \sum_{\alpha=1}^{n} u^{\alpha \bar{\beta}} u_{x_{n} \alpha}=2 \delta_{\beta n}-i \sum_{\alpha=1}^{n} u^{\alpha \bar{\beta}} u_{y_{n} \alpha}, \\
& \sum_{\beta=1}^{n} u^{\alpha \bar{\beta}} u_{x_{n} \bar{\beta}}=2 \delta_{\alpha n}+i \sum_{\beta=1}^{n} u^{\alpha \bar{\beta}} u_{y_{n} \bar{\beta}} .
\end{aligned}
$$

Then

$$
\begin{aligned}
& L\left(-\left(u_{y_{n}}-\underline{u}_{y_{n}}\right)^{2} \pm T_{j}(u-\underline{u})\right) \\
& \geq-C_{9}\left(1+\sum u^{\alpha \bar{\alpha}}\right)+2 \sum u^{\alpha \bar{\beta}}\left(u_{y_{n}}-\underline{u}_{y_{n}}\right)_{\alpha}\left(u_{y_{n}}-\underline{u}_{y_{n}}\right)_{\bar{\beta}} \\
& \mp \sum u^{\alpha \bar{\beta}}\left(\left(u_{y_{n}}-\underline{u}_{y_{n}}\right)_{\alpha}\left(-i P_{s_{j}}\right)_{\bar{\beta}}+\left(u_{y_{n}}-\underline{u}_{y_{n}}\right)_{\bar{\beta}}\left(i P_{s_{j}}\right)_{\alpha}\right)
\end{aligned}
$$


where $C_{9}>0$ depend on $\epsilon, C_{1}, C_{2}, C_{3}, M, C_{\varphi}, C_{f}, \rho, P$.

By the Cauchy-Schwarz inequality,

$2 \sum u^{\alpha \bar{\beta}}\left(u_{y_{n}}-\underline{u}_{y_{n}}\right)_{\alpha}\left(u_{y_{n}}-\underline{u}_{y_{n}}\right)_{\bar{\beta}}+\frac{1}{2} \sum u^{\alpha \bar{\beta}}\left(i P_{s_{j}}\right)_{\alpha}\left(-i P_{s_{j}}\right)_{\bar{\beta}}$

$\geq \pm \sum u^{\alpha \bar{\beta}}\left(\left(u_{y_{n}}-\underline{u}_{y_{n}}\right)_{\alpha}\left(-i P_{s_{j}}\right)_{\bar{\beta}}+\left(u_{y_{n}}-\underline{u}_{y_{n}}\right)_{\bar{\beta}}\left(i P_{s_{j}}\right)_{\alpha}\right)$.

Then

$$
L\left(-\left(u_{y_{n}}-\underline{u}_{y_{n}}\right)^{2} \pm T_{j}(u-\underline{u})\right) \geq-C_{10}\left(1+\sum u^{\alpha \bar{\alpha}}\right),
$$

where $C_{10}>0$ depends on $\Omega, C_{\varphi}, C_{f}, \epsilon, T, M$.

Hence, by Lemma 4.2, we can choose $A, B>0$ independent of $u$ so that

$$
\begin{gathered}
L\left(\psi_{ \pm}\right) \geq 0 \quad \text { on } \quad \Omega_{\delta} \times(\epsilon, T), \\
\psi_{ \pm} \geq 0 \quad \text { on } \quad \partial_{P}\left(\Omega_{\delta} \times(\epsilon, T)\right) .
\end{gathered}
$$

By the maximum principle, we obtain $\psi_{ \pm} \geq 0$ on $\Omega_{\delta} \times\left(\frac{\epsilon}{2}, T\right)$.

Note that $\psi_{ \pm}(p, t)=0$ for $t \in\left(\frac{\epsilon}{2}, T\right)$.

Hence,

$$
\lim _{x_{n} \searrow 0} \frac{\psi_{ \pm}\left(p+\left(0, \ldots, x_{n}\right), t\right)-\psi_{ \pm}(p, t)}{x_{n}} \geq 0
$$

thus

$$
\left|u_{s_{j} x_{n}}(p, t)\right| \leq D_{1}^{\prime \prime},
$$

where $t \in(\epsilon, T)$ and $D^{\prime \prime}{ }_{1}>0$ depend only on $\Omega, C_{\varphi}, C_{f}, \epsilon, T, C_{u}$.

Step 3:Bounding the normal-normal derivatives.

We have that

$$
\operatorname{det}\left(u_{\alpha \bar{\beta}}\right)=e^{\dot{u}-f(t, z, u)}
$$

is bounded from above and below on $\partial \Omega \times(\epsilon, T)$.

By step 1 and step 2, $\left|u_{z_{n} \bar{z}_{n}} \operatorname{det}\left(u_{\alpha \bar{\beta}}\right)_{\alpha, \beta \leq n-1}\right|$ is bounded on $\{p\} \times(\epsilon, T)$.

Hence, by Lemma 4.2, we obtain

$$
\left|u_{z_{n} \bar{z}_{n}}(p, t)\right| \leq D_{1}^{\prime \prime \prime}, t \in(\epsilon, T),
$$

where $D_{1}^{\prime \prime \prime}$ depends on $\Omega, C_{\varphi}, C_{f}, \epsilon, T, C_{u}$.

Consequently

$$
\left|u_{x_{n} x_{n}}\right| \leq D_{1}^{\prime \prime \prime \prime},
$$

where $D_{1}^{\prime \prime \prime \prime}$ depends on $\Omega, C_{\varphi}, C_{f}, \epsilon, T, C_{u}$.

\subsection{Interior estimate of the Laplacian.}

Proposition 4.4. There exists $D_{2}=D_{2}\left(\Omega, \epsilon, T, C_{\varphi}, C_{f}, C_{u}\right)$ such that

$$
\Delta u \leq D_{2} \quad \text { on } \quad \Omega \times(\epsilon, T) .
$$

Proof. We set

$$
\phi=(t-\epsilon) \log \Delta u+A_{1}|z|^{2}-A_{2} t,
$$

where $A_{1}, A_{2}>0$ will be specified later.

We have 


$$
\begin{aligned}
L(\phi) & =\log \Delta u+(t-\epsilon) \frac{\Delta \dot{u}}{\Delta u}-A_{2}-(t-\epsilon) \sum u^{\alpha \bar{\beta}}(\log \Delta u)_{\alpha \bar{\beta}} \\
& -A_{1} \sum u^{\alpha \bar{\alpha}}-\phi f_{u}(t, z, u) .
\end{aligned}
$$

By Theorem 2.7,

$$
\log \Delta u \leq \log n+\log \operatorname{det}\left(u_{\alpha \bar{\beta}}\right)+(n-1) \log \left(\sum u^{\alpha \bar{\alpha}}\right) .
$$

By Theorem 2.8,

$$
\begin{aligned}
\frac{\Delta \dot{u}}{\Delta u}-\sum u^{\alpha \bar{\beta}}(\log \Delta u)_{\alpha \bar{\beta}} & \leq \frac{\Delta \dot{u}}{\Delta u}-\frac{\Delta \log \operatorname{det}\left(u_{\alpha \bar{\beta}}\right)}{\Delta u} \\
& =\frac{\Delta f(t, z, u)}{\Delta u} \\
& =\frac{\Delta_{z} f(t, z, u)}{\Delta u}+f_{u}(t, z, u)+\sum \frac{f_{u s_{j}}(t, z, u) u_{s_{j}}}{\Delta u} \\
& +\sum \frac{f_{u u}(t, z, u) u_{s_{j}}^{2}}{\Delta u} .
\end{aligned}
$$

Hence, there exist $A_{1}, A_{2}>0$ depending on $\Omega, \epsilon, T, C_{\varphi}, C_{f}, C_{u}$ such that

$$
L(\phi) \leq 0 \text { on } \Omega \times(\epsilon, T) .
$$

Thus, by the maximum principle and Proposition 4.3.

$$
(t-\epsilon) \log \Delta u \leq D_{2}^{\prime} \quad \text { on } \quad \Omega \times(\epsilon, T),
$$

where $D_{2}^{\prime}$ depends on $\Omega, \epsilon, T, C_{\varphi}, C_{f}, C_{u}$.

Therefore,

$$
\Delta u \leq e^{D_{2}^{\prime} / \epsilon} \quad \text { on } \quad \Omega \times(2 \epsilon, T) .
$$

5. $C^{2, \alpha}$ ESTimate UP TO THE Boundary FOR THE PARABOliC EQUATION

\subsection{Parabolic Hölder spaces.}

The reader can find more complete notations in [Lieb96, Chapter 4] or [Kryl96. Chapter 8].

In $\mathbb{R}^{N} \times \mathbb{R}$ we define the parabolic distance between the points $X_{1}=\left(x_{1}, t_{1}\right), X_{2}=$ $\left(x_{2}, t_{2}\right)$ as

$$
d\left(X_{1}, X_{2}\right)=\left|x_{1}-x_{2}\right|+\left|t_{1}-t_{2}\right|^{1 / 2} .
$$

Let $0<\alpha<1$. Let $u$ be a function defined in a domain $Q \subset \mathbb{R}^{N} \times \mathbb{R}$. We say that $u$ is uniformly Hölder continuous in $Q$ with exponent $\alpha$, or $u \in C^{\alpha}(Q)$, if and only if

$$
[u]_{\alpha ; Q}=\sup _{X_{j} \in Q, X_{1} \neq X_{2}} \frac{\left|u\left(X_{1}\right)-u\left(X_{2}\right)\right|}{d^{\alpha}\left(X_{1}, X_{2}\right)}<\infty .
$$

Let $0<\beta<2$. We denote 


$$
\langle u\rangle_{\beta ; Q}=\sup _{\left(x, t_{1}\right) \neq\left(x, t_{2}\right) \in Q} \frac{\left|u\left(x, t_{1}\right)-u\left(x, t_{2}\right)\right|}{\left|t_{1}-t_{2}\right|^{\beta / 2}} .
$$

We say that $u$ is uniformly Hölder continuous in $Q$ with exponent $k+\alpha$, or $u \in C^{k, \alpha}(Q)$ if the derivatives $D_{x}^{j} D_{t}^{l} u$ exist for $|j|+2 l \leq k$ and the norm

$$
\|u\|_{C^{k, \alpha}(Q)}=\sum_{|j|+2 l \leq k} \sup _{Q}\left|D_{x}^{j} D_{t}^{l} u\right|+\sum_{|j|+2 l=k}\left[D_{x}^{j} D_{t}^{l} u\right]_{\alpha ; Q}+\sum_{|j|+2 l=k-1}\left\langle D_{x}^{j} D_{t}^{l} u\right\rangle_{\alpha+1 ; Q}
$$

is finite.

The norm $\|\cdot\|_{C^{k, \alpha}(Q)}$ makes $C^{k, \alpha}(Q)$ a Banach space. If we define the similar notions for $\bar{Q}$, then $C^{k, \alpha}(Q)=C^{k, \alpha}(\bar{Q})$.

\section{2. $\mathrm{C}^{2, \alpha}$ estimate up to the boundary.}

Let $\Omega$ be a bounded smooth domain of $\mathbb{R}^{N}$. We consider the equation

$$
\dot{u}=F\left(D^{2} u\right)+f(t, x, u) \text { in } \Omega \times(0, \tilde{T}),
$$

where $\tilde{T}>0, f$ is a smooth function defined on $[0, \tilde{T}) \times \bar{\Omega} \times \mathbb{R}$ and $F$ is a smooth concave function defined on the set of all real $N \times N$ matrices. In addition, we assume that there exist $0<\lambda<\Lambda<\infty$ such that

$$
\lambda \operatorname{tr} \eta \leq F(r+\eta)-F(r) \leq \Lambda \operatorname{tr} \eta
$$

for any symmetric matrix $r$, any positive definite matrix $\eta$.

We will establish $C^{2, \alpha}$ estimates for the solution of (27) on $\bar{\Omega} \times(\epsilon, T)$ for any $0<\epsilon<$ $T<\tilde{T}$ without $C^{2, \alpha}$ conditions on $\Omega \times\{0\}$. The main result of this section is the following:

Theorem 5.1. Let $F$ be concave and smooth satisfying (28). Let $f$ be a smooth function in $[0, \tilde{T}) \times \bar{\Omega} \times \mathbb{R}$ and $\varphi$ be a smooth function in $\bar{\Omega} \times[0, \tilde{T})$. Assume that $u \in C^{2 ; 1}(\bar{\Omega} \times[0, \tilde{T})) \cap C^{\infty}(\Omega \times(0, \tilde{T}))$ is a solution of

$$
\begin{cases}\dot{u}=F\left(D^{2} u\right)+f(t, x, u) & \text { in } \Omega \times(0, \tilde{T}), \\ u=\varphi & \text { on } \partial \Omega \times(0, \tilde{T}),\end{cases}
$$

and that

$$
|u|+|\dot{u}|+|\nabla u|+\left|D^{2} u\right| \leq C,
$$

then $u \in C^{2, \alpha}(\bar{\Omega} \times(0, \tilde{T}))$ satisfies

$$
\|u\|_{C^{2, \alpha}(\Omega \times(\epsilon, T))} \leq C_{\epsilon, T} \quad \forall 0<\epsilon<T<\tilde{T},
$$

where $0<\alpha<1, C_{\epsilon, T}>0$ depend on $\lambda, \Lambda, \Omega, C, \epsilon, T$ and the upper bound of $\|\varphi\|_{C^{4}}+$ $\|F\|_{C^{1}}+\|f\|_{C^{2}}$.

Remark 5.2. In the theorem above, we denote

$$
\begin{gathered}
\|\varphi\|_{C^{k}(\Omega \times(0, \tilde{T}))}=\sum_{|j|+2 l \leq k} \sup _{\Omega \times(0, \tilde{T})}\left|D_{x}^{j} D_{t}^{l} \varphi\right|, \\
\|F\|_{C^{k}(\operatorname{Mat}(N \times N, \mathbb{R}))}=\sum_{|j| \leq k} \sup \left|D^{j} F\right|
\end{gathered}
$$




$$
\|f\|_{\left.C^{k}((0, \tilde{T}) \times \Omega \times \mathbb{R})\right)}=\sum_{j_{1}+\left|j_{2}\right|+j_{3} \leq k} \sup \left|D_{t}^{j_{1}} D_{x}^{j_{2}} D_{u}^{j_{3}} f\right| .
$$

In order to prove Theorem [5.1, we use the technique of Caffarelli as in [CC95]. We need to prove a series of lemmas.

Lemma 5.3. There exist $0<\beta<1$ and $C_{\epsilon, T}>0$ depending on $\lambda, \Lambda, \Omega, C, \epsilon, T$ and the upper bound of $\|\varphi\|_{C^{4}}+\|F\|_{C^{1}}+\|f\|_{C^{1}}$ such that

$$
\frac{\left\|D^{2} u(x, t)-D^{2} u\left(x_{0}, t_{0}\right)\right\|}{\left(\left|x-x_{0}\right|+\left|t-t_{0}\right|^{1 / 2}\right)^{\beta}} \leq C_{\epsilon, T}, \quad \forall x, x_{0} \in \partial \Omega ; \forall t, t_{0} \in(\epsilon, T) .
$$

Proof. Let $x_{0} \in \partial \Omega$. We consider a smooth diffeomorphism

$$
\begin{aligned}
\psi: U \cap \Omega \longrightarrow B_{4}^{+} & :=\left\{y \in \mathbb{R}^{N}:|y|<4, y_{N}>0\right\} \\
x & \mapsto y=\psi(x)
\end{aligned}
$$

such that $\psi\left(x_{0}\right)=0$ and

$$
\psi(U \cap \partial \Omega)=\Gamma_{4}=\left\{y=\left(y^{\prime}, y_{N}\right) \in \mathbb{R}^{N-1} \times \mathbb{R}:\left|y^{\prime}\right|<4, y_{N}=0\right\},
$$

where $U$ is a neighborhood of $x_{0}$.

We define

$$
v(y, t)=u\left(\psi^{-1}(y), t\right)-\varphi\left(\psi^{-1}(y), t\right),
$$

where $y \in B_{4}^{+} \bigcup \Gamma_{4}, t \in(\epsilon, T)$. Then $\left.v\right|_{\Gamma_{4} \times(\epsilon, T)}=0$ and $v$ satisfies the equation

$$
\dot{v}=G\left(t, y, v, D v, D^{2} v\right)
$$

where the upper bound of $\|G\|_{C^{1}}$ depends on $\|F\|_{C^{1}},\|f\|_{C^{1}}$ and $\psi$. Moreover,there exists $A>1$ depending on $\psi$ (hence, $A$ depends only on $\Omega$ ) such that

$$
\frac{\lambda}{A}|\xi|^{2} \leq \frac{\partial G}{\partial r_{i j}} \xi_{i} \xi_{j} \leq A \Lambda|\xi|^{2}
$$

for all $\xi \in \mathbb{R}^{N}$.

Now we only need to show

$$
\left\|D^{2} v(y, t)-D^{2} v\left(0, t_{0}\right)\right\| \leq C_{\epsilon, T}\left(|y|+\left|t-t_{0}\right|^{1 / 2}\right)^{\beta}
$$

for any $y \in \Gamma_{1}, t, t_{0} \in(\epsilon, T)$.

By the implicit function theorem, we have

$$
v_{N N}=H\left(t, y, v, \dot{v}, D v,\left(v_{i j}\right)_{j<N}\right) .
$$

By the chain rule, we have

$$
|D H| \leq \frac{A}{\lambda}(\sup |D G|+1) .
$$

Hence, there exists $B>0$ such that

$$
\begin{aligned}
\left|v_{N N}(y, t)-v_{N N}\left(0, t_{0}\right)\right| & \leq B\left(\sup _{j<N}\left|v_{i j}(y, t)-v_{i j}\left(0, t_{0}\right)\right|+\left|\dot{v}(y, t)-\dot{v}\left(0, t_{0}\right)\right|\right. \\
& \left.+\left|D v(y, t)-D v\left(0, t_{0}\right)\right|+|y|+\left|t-t_{0}\right|\right) .
\end{aligned}
$$


Note that $\left.\dot{v}\right|_{\Gamma_{4} \times(\epsilon, T)}=\left.v_{j}\right|_{\Gamma_{4} \times(\epsilon, T)}=\left.v_{i j}\right|_{\Gamma_{4} \times(\epsilon, T)}=0$ for $j<N$. Then we only need to show

$$
\begin{gathered}
\left|v_{N}(y, t)-v_{N}\left(0, t_{0}\right)\right| \leq C_{\epsilon, T}\left(|y|+\left|t-t_{0}\right|^{1 / 2}\right)^{\beta}, \\
\left|v_{N k}(y, t)-v_{N k}\left(0, t_{0}\right)\right| \leq C_{\epsilon, T}\left(|y|+\left|t-t_{0}\right|^{1 / 2}\right)^{\beta},
\end{gathered}
$$

for any $y \in \Gamma_{1}, t, t_{0} \in(\epsilon, T)$ and $k<N$.

By (31), we have

$$
\dot{v}=\Delta v+f_{1}(t, y)
$$

where $\Delta$ is the Laplacian operator and $f_{1}(t, y)=G\left(t, y, v, D v, D^{2} v\right)-\Delta v$. By the hypothesis of theorem, $\left\|f_{1}\right\|_{L^{\infty}}$ is bounded by a universal constant.

Now we take the derivative of equation (31) in the direction $y_{k}$ and get that

$$
\dot{v}_{k}=\sum_{i, j=1}^{N}\left(v_{k}\right)_{i j} \frac{\partial G}{\partial r_{i j}}\left(t, y, v, D v, D^{2} v\right)+f_{2}(t, y),
$$

where

$$
f_{2}(t, y)=\frac{\partial G}{\partial y_{k}}\left(t, y, v, D v, D^{2} v\right)+v_{k} \frac{\partial G}{\partial p}\left(t, y, v, D v, D^{2} v\right)+\sum_{l=1}^{N} v_{l k} \frac{\partial G}{\partial q_{l}}\left(t, y, v, D v, D^{2} v\right) .
$$

Then $\left\|f_{2}\right\|_{L^{\infty}}$ is bounded by a universal constant.

Then [Lieb96, Lemma 7.32] states that

Lemma 5.4. If $u \in C^{2 ; 1}\left(B_{4}^{+} \times(0, T)\right)$ satisfies

$$
\begin{aligned}
& \left|\dot{u}-\sum a_{i j} u_{i j}\right| \leq A_{1}, \\
& |u| \leq A_{2} x_{N},
\end{aligned}
$$

where $a_{i j} \in C\left(B_{4}^{+} \times(0, T)\right)$ is such that

$$
\begin{gathered}
\sup \left|a_{i j}\right| \leq B \text { and } \\
\lambda|\xi|^{2} \leq \sum a_{i j} \xi_{i} \xi_{j} \leq \Lambda|\xi|^{2},
\end{gathered}
$$

then there are positive constants $\beta$ and $C$ determined only by $A_{1}, A_{2}, B, \lambda, \Lambda, \epsilon, T, N$ such that

$$
\left(\sup _{U(y, t, R)} \frac{u}{x_{N}}-\inf _{U(y, R)} \frac{u}{x_{N}}\right) \leq C R^{\beta}\left(\sup _{B_{4}^{+} \times(0, T)} \frac{u}{x_{N}}-\inf _{B_{4}^{+} \times(0, T)} \frac{u}{x_{N}}+1\right),
$$

where $y \in B_{1}^{+}, 2 \epsilon<t<T-2 \epsilon, R<\epsilon$ and $U(y, t, R)=B_{R}^{+}(y) \times\left(t-R^{2}, t+R^{2}\right)$.

Applying this lemma to the equations (34) and (35), we obtain (32) and (33).

Corollary 5.5. There exists $C_{\epsilon, T}>0$ depending on $\lambda, \Lambda, \Omega, C, \epsilon, T$ and the upper bound of $\|\varphi\|_{C^{4}}+\|F\|_{C^{1}}+\|f\|_{C^{1}}$ such that

$$
\frac{\left|\dot{u}(x, t)-\dot{u}\left(x_{0}, t_{0}\right)\right|}{\left(\left|x-x_{0}\right|+\left|t-t_{0}\right|^{1 / 2}\right)^{\beta}} \leq C_{\epsilon, T}, \quad \forall x, x_{0} \in \partial \Omega ; \forall t, t_{0} \in(\epsilon, T) .
$$

where $0<\beta<1$ is the constant in Lemma 5.3. 
Lemma 5.6. There exists $C_{\epsilon, T}>0$ depending on $\lambda, \Lambda, \Omega, C, \epsilon, T$ and the upper bound of $\|\varphi\|_{C^{4}}+\|F\|_{C^{1}}+\|f\|_{C^{1}}$ such that

$$
\frac{\left|\dot{u}(x, t)-\dot{u}\left(x_{0}, t_{0}\right)\right|}{\left(\left|x-x_{0}\right|+\left|t-t_{0}\right|^{1 / 2}\right)^{\beta / 2}} \leq C_{\epsilon, T}, \quad \forall x \in \Omega, x_{0} \in \partial \Omega ; \forall t, t_{0} \in(\epsilon, T) .
$$

where $0<\beta<1$ is the constant in Lemma 5.3.

Proof. By equation (29), we have

$$
\left|\ddot{u}-\sum \frac{\partial F}{\partial r_{i j}} \dot{u}_{i j}\right|=\left|f_{t}(t, x, u)+\dot{u} f_{u}(t, x, u)\right| \leq A,
$$

where $A>0$ is a universal constant.

Let $x_{0} \in \partial \Omega$ and $t_{0} \in(2 \epsilon, T)$. We can choose coordinates $\left(x_{j}\right)_{1 \leq j \leq N}$ so that $x_{0}=0$ and the positive $x_{N}$ axis is the interior normal direction of $\partial \Omega$ at $x_{0}$. We also assume that near $x_{0}, \partial \Omega$ is represented as a graph

$$
x_{N}=P\left(x^{\prime}\right)=\sum_{j, k<N} P_{j k} x_{j} x_{k}+O\left(\left|x^{\prime}\right|^{3}\right),
$$

where $x^{\prime}=\left(x_{1}, \ldots, x_{N-1}\right)$.

Let $Q\left(x^{\prime}\right)=P\left(x^{\prime}\right)-\left|x^{\prime}\right|^{2}$. We consider

$$
v=K_{1}\left(x_{N}-Q\left(x^{\prime}\right)\right)^{\beta / 2}+K_{2}\left(\left(x_{N}-Q\left(x^{\prime}\right)\right)^{2}+\left(t_{0}-t\right)\right)^{\beta / 4} \text {. }
$$

We have

$$
\begin{aligned}
\frac{\partial^{2}\left(x_{N}-Q\left(x^{\prime}\right)\right)^{\beta / 2}}{\partial x_{i} \partial x_{j}} & =\frac{\beta(\beta-2)}{4}\left(x_{N}-Q\left(x^{\prime}\right)\right)^{\beta / 2-2} \frac{\partial\left(x_{N}-Q\left(x^{\prime}\right)\right)}{\partial x_{i}} \frac{\partial\left(x_{N}-Q\left(x^{\prime}\right)\right)}{\partial x_{j}} \\
& +\frac{\beta}{2}\left(x_{N}-Q\left(x^{\prime}\right)\right)^{\beta / 2-1} \frac{\partial^{2}\left(x_{N}-Q\left(x^{\prime}\right)\right)}{\partial x_{i} \partial x_{j}},
\end{aligned}
$$

and

$$
\begin{aligned}
& \frac{\partial^{2}\left(\left(x_{N}-Q\left(x^{\prime}\right)\right)^{2}+t_{0}-t\right)^{\beta / 4}}{\partial x_{i} \partial x_{j}} \\
& =\frac{\beta(\beta-4)}{4}\left(\left(x_{N}-Q\left(x^{\prime}\right)\right)^{2}+t_{0}-t\right)^{\beta / 4-2}\left(x_{N}-Q\left(x^{\prime}\right)\right)^{2} \frac{\partial\left(x_{N}-Q\left(x^{\prime}\right)\right)}{\partial x_{i}} \frac{\partial\left(x_{N}-Q\left(x^{\prime}\right)\right)}{\partial x_{j}} \\
& +\frac{\beta}{4}\left(\left(x_{N}-Q\left(x^{\prime}\right)\right)^{2}+t_{0}-t\right)^{\beta / 4-1} \frac{\partial^{2}\left(x_{N}-Q\left(x^{\prime}\right)\right)^{2}}{\partial x_{i} \partial x_{j}} .
\end{aligned}
$$

Hence, there exists $R>0$ satisfying, by $F_{r_{11}} \geq \lambda$,

$$
\sum_{i, j=1}^{N} \frac{\partial F}{\partial r_{i j}} \frac{\partial^{2}\left(x_{N}-Q\left(x^{\prime}\right)\right)^{\beta / 2}}{\partial x_{i} \partial x_{j}} \leq \frac{\lambda \beta(\beta-2)}{6}\left(x_{N}-Q\left(x^{\prime}\right)\right)^{\beta / 2-2}<0,
$$

and

$$
\sum_{i, j=1}^{N} \frac{\partial F}{\partial r_{i j}} \frac{\partial^{2}\left(\left(x_{N}-Q\left(x^{\prime}\right)\right)^{2}+t_{0}-t\right)^{\beta / 4}}{\partial x_{i} x_{j}}=O\left(x_{N}-Q\left(x^{\prime}\right)\right)^{\beta / 2-2} .
$$

On the other hand,

$$
\left.\left|\dot{u}-\dot{u}\left(0, t_{0}\right)\right|\right|_{\partial_{P}\left(\left(\Omega \cap B_{R}\right) \times\left(\epsilon, t_{0}\right)\right)}=O\left(\left(\left(x_{N}-Q\left(x^{\prime}\right)\right)^{2}+t_{0}-t\right)^{\beta / 4}\right) .
$$


By (36), (37), (38), (39), there exists $K_{1}, K_{2}>0$ such that

$$
\begin{gathered}
\left.v\right|_{\partial_{P}\left(\left(\Omega \cap B_{R}\right) \times\left(\epsilon, t_{0}\right)\right)} \geq \pm\left.\left(\dot{u}-\dot{u}\left(0, t_{0}\right)\right)\right|_{\partial_{P}\left(\left(\Omega \cap B_{R}\right) \times\left(\epsilon, t_{0}\right)\right)}, \\
( \pm \ddot{u}-\dot{v})-\sum \frac{\partial F}{\partial r_{i j}}\left( \pm \dot{u}_{i j}-v_{i j}\right) \leq A+\frac{K_{1} \lambda \beta(\beta-2)}{8} \leq 0 .
\end{gathered}
$$

The comparison principle of parabolic type ([Fried83] $)$ states that

Lemma 5.7. Let $\Omega$ be a bounded domain of $\mathbb{R}^{N}$ and $T>0$. Let $u, v \in C^{2 ; 1}(\Omega \times$ $(0, T]) \cap C(\bar{\Omega} \times[0, T])$. Assume that

$$
\frac{\partial(u-v)}{\partial t}-\sum a_{i j} \frac{\partial^{2}(u-v)}{\partial x_{i} \partial x_{j}}-b \cdot(u-v) \leq 0,
$$

where $a_{i j}, b \in C(\Omega \times(0, T)),\left(a_{i j}(x, t)\right)$ are positive definite symmetric matrices and $b(z, t)<0$. Then $(u-v) \leq \max \left(0, \sup _{\partial_{P}(\Omega \times(0, T))}(u-v)\right)$.

Applying the comparison principle, we have

$$
\left.\left(\dot{u}-\dot{u}\left(0, t_{0}\right)\right)\right|_{\left(\Omega \cap B_{R}\right) \times\left(\epsilon, t_{0}\right)} \leq\left. v\right|_{\left(\Omega \cap B_{R}\right) \times\left(\epsilon, t_{0}\right)} .
$$

Hence there exists $K>0$ such that

$$
\left|\dot{u}(x, t)-\dot{u}\left(0, t_{0}\right)\right| \leq K\left(|x|+\left|t-t_{0}\right|^{1 / 2}\right)^{\beta / 2},
$$

where $x \in \Omega \times B_{R}$ and $\epsilon<t \leq t_{0}$.

Note that $R$ is independent of $x_{0}$ and $K$ is independent of $t_{0}$. Then there exists $C_{\epsilon, T}$ such that

$$
\frac{\left|\dot{u}(x, t)-\dot{u}\left(x_{0}, t_{0}\right)\right|}{\left(\left|x-x_{0}\right|+\left|t-t_{0}\right|^{1 / 2}\right)^{\beta / 2}} \leq C_{\epsilon}, \quad \forall x \in \Omega, x_{0} \in \partial \Omega ; \forall t, t_{0} \in(2 \epsilon, T) .
$$

Lemma 5.8. There exists $C_{\epsilon, T}>0$ depending on $\lambda, \Lambda, \Omega, C, \epsilon, T$ and upper bound of $\|\varphi\|_{C^{4}}+\|F\|_{C^{1}}+\|f\|_{C^{2}}$ such that

$$
u_{\xi \xi}(x, t)-u_{\xi \xi}\left(x_{0}, t_{0}\right) \leq C_{\epsilon, T}\left(\left|x-x_{0}\right|+\left|t-t_{0}\right|^{1 / 2}\right)^{\beta / 2}
$$

for any $\xi \in \mathbb{R}^{N},|\xi|=1, x \in \Omega, x_{0} \in \partial \Omega, \epsilon<t, t_{0}<T$. Where $0<\beta<1$ is the constant in Lemma 5.3 .

Proof. By the equation (29), we have

$$
\dot{u}_{\xi \xi}-\sum \frac{\partial F}{\partial r_{i j}}\left(u_{\xi \xi}\right)_{i j}-f_{u} . u_{\xi \xi}=\sum \frac{\partial^{2} F}{\partial r_{i j} \partial r_{k l}}\left(u_{\xi}\right)_{i j}\left(u_{\xi}\right)_{k l}+O(1) \leq O(1)
$$

By Lemma 5.3, we also obtain

$$
\left.\left.\left(u_{\xi \xi}(x, t)-u_{\xi \xi}\left(x_{0}, t_{0}\right)\right)\right|_{\partial_{P}(\Omega \times(\epsilon, T))}=O\left(\left|x-x_{0}\right|+\left|t-t_{0}\right|^{1 / 2}\right)^{\beta / 2}\right)
$$

Then, the proof of Lemma 5.8 is similar to the proof of Lemma 5.6 with the same type of fuction $v$. 
Lemma 5.9. There exists $C_{\epsilon, T}>0$ depending on $\lambda, \Lambda, \Omega, C, \epsilon, T$ and upper bound of $\|\varphi\|_{C^{4}}+\|F\|_{C^{1}}+\|f\|_{C^{2}}$ such that

$$
\left\|D^{2} u(x, t)-D^{2} u\left(x_{0}, t_{0}\right)\right\| \leq C_{\epsilon, T}\left(\left|x-x_{0}\right|+\left|t-t_{0}\right|^{1 / 2}\right)^{\beta / 2}
$$

for any $x \in \Omega, x_{0} \in \partial \Omega, \epsilon<t, t_{0}<T$, where $0<\beta<1$ is the constant in Lemma 5.3. Proof. Let $\lambda_{1}, \ldots, \lambda_{N}$ be eigenvalues of $D^{2} u(x, t)-D^{2} u\left(x_{0}, t_{0}\right)$. We have

Moreover,

$$
\left\|D^{2} u(x, t)-D^{2} u\left(x_{0}, t_{0}\right)\right\| \leq \sum\left|\lambda_{i}\right| .
$$

$$
\begin{aligned}
\dot{u}(x, t)-f(t, x, u(x, t)) & =F\left(D^{2} u(x, t)\right) \\
& \leq F\left(D^{2} u\left(x_{0}, t_{0}\right)\right)+\Lambda \sum_{\lambda_{i}>0} \lambda_{i}+\lambda \sum_{\lambda_{i}<0} \lambda_{i} \\
& =\dot{u}\left(x_{0}, t_{0}\right)-f\left(t_{0}, x_{0}, u\left(x_{0}, t_{0}\right)\right)+\Lambda \sum_{\lambda_{i}>0} \lambda_{i}+\lambda \sum_{\lambda_{i}<0} \lambda_{i} .
\end{aligned}
$$

Hence, by Lemma 5.6 , we have

$$
\Lambda \sum_{\lambda_{i}>0}\left|\lambda_{i}\right| \geq \lambda \sum_{\lambda_{i}<0}\left|\lambda_{i}\right|-A\left(\left|x-x_{0}\right|+\left|t-t_{0}\right|^{1 / 2}\right)^{\beta / 2}
$$

where $A>0$ is a universal constant.

Then

$$
\left\|D^{2} u(x, t)-D^{2} u\left(x_{0}, t_{0}\right)\right\| \leq \frac{\Lambda+\lambda}{\lambda} \sum_{\lambda_{i}>0}\left|\lambda_{i}\right|+\frac{A}{\lambda}\left(\left|x-x_{0}\right|+\left|t-t_{0}\right|^{1 / 2}\right)^{\beta / 2} .
$$

Note that

$$
\sum_{\lambda_{i}>0}\left|\lambda_{i}\right| \leq N \max \left\{0, \lambda_{1}, \ldots \lambda_{N}\right\} \leq N \max \left\{\sup _{|\xi|=1}\left(u_{\xi \xi}(x, t)-u_{\xi \xi}\left(x_{0}, t_{0}\right)\right), 0\right\} .
$$

By Lemma 5.8, there exists $C_{\epsilon, T}>0$ depending on $\lambda, \Lambda, \Omega, C, \epsilon, T$ and upper bound of $\|\varphi\|_{C^{4}}+\|F\|_{C^{1}}+\|f\|_{C^{2}}$ such that

$$
\left\|D^{2} u(x, t)-D^{2} u\left(x_{0}, t_{0}\right)\right\| \leq C_{\epsilon, T}\left(\left|x-x_{0}\right|+\left|t-t_{0}\right|^{1 / 2}\right)^{\beta / 2}
$$

for any $x \in \Omega, x_{0} \in \partial \Omega, \epsilon<t, t_{0}<T$.

Proof of Theorem 5.1. We need to show that

$$
\left\|D^{2} u\left(x, t_{1}\right)-D^{2} u\left(y, t_{2}\right)\right\| \leq C\left(|x-y|+\left|t_{1}-t_{2}\right|^{1 / 2}\right)^{\gamma},
$$

where $x, y \in \Omega, 2 \epsilon<t_{1}, t_{2}<T-\epsilon . C$ and $\gamma$ are universal constants.

We can assume that $d_{x}:=d(x, \partial \Omega) \geq d_{y}:=d(y, \partial \Omega)$.

If $\left.|x-y|^{2}+\left|t_{1}-t_{2}\right| \leq \min \left\{\frac{d_{x}^{2}}{4}, \frac{\epsilon}{2}\right\}\right)$, we denote

$$
v(\xi, t)=\frac{1}{a^{2}}\left(u\left(x+a . \xi, t_{1}+a^{2} t\right)-u\left(x, t_{1}\right)-a \sum u_{k}\left(x, t_{1}\right) \xi_{k}\right),
$$

where $a=\min \left\{d_{x}, \epsilon^{1 / 2}\right\}$. Then $v \in C^{\infty}(\mathbb{B} \times(-1,1))$ satisfies

$$
\dot{v}=F\left(D^{2} v\right)+f\left(t_{1}+a^{2} t, x_{1}+a \xi, u\left(x_{1}+a \xi, t_{1}+a^{2} t\right)\right)=F\left(D^{2} v\right)+\tilde{f}(t, \xi) .
$$


It follows from the interior estimate (see the theorem 14.7 and the lemma 14.8 of [Lieb96]) that

$$
\|v\|_{C^{2, \gamma}\left(\mathbb{B}_{1 / 2} \times(-1 / 2,1 / 2)\right)} \leq A\left(\|v\|_{C^{2}(\mathbb{B} \times(-1,1))}+1\right),
$$

where $A$ is universal, $\gamma=\min \{\alpha, \beta / 2\}, \beta$ is the constant in Lemma 5.3 and $\alpha$ is the constant in Theorem 14.7 of [Lieb96].

Moreover

$$
\begin{aligned}
&|v(\xi, t)| \leq \frac{\left|u\left(x+a \xi, t_{1}+a^{2} t\right)-u\left(x+a \xi, t_{1}\right)\right|}{a^{2}} \\
&+\frac{\left|u\left(x+a \xi, t_{1}\right)-u\left(x, t_{1}\right)-a \sum u_{k}\left(x, t_{1}\right) \xi_{k}\right|}{a^{2}} \\
& \leq \sup |\dot{u}|+\sup \left\|D^{2} u\right\|, \\
&|\dot{v}(\xi, t)|=\left|\dot{u}\left(x+a \xi, t_{1}+a^{2} t\right)\right| \leq \sup |\dot{u}|, \\
&\left\|D^{2} v(\xi, t)\right\|=\left\|D^{2} u\left(x+a \xi, t_{1}+a^{2} t\right)\right\| \leq \sup \left\|D^{2} u\right\| .
\end{aligned}
$$

Hence

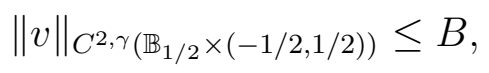

where $B$ is universal.

Then

$$
\left\|D^{2} u\left(x, t_{1}\right)-D^{2} u\left(y, t_{2}\right)\right\| \leq B\left(|x-y|+\left|t_{1}-t_{2}\right|^{1 / 2}\right)^{\gamma} .
$$

If $|x-y|^{2}+\left|t_{1}-t_{2}\right| \geq \frac{\epsilon}{2}$, then

$$
\left\|D^{2} u\left(x, t_{1}\right)-D^{2} u\left(y, t_{2}\right)\right\| \leq 2\left(\frac{\epsilon}{2}\right)^{-\gamma / 2}\left(\sup \left\|D^{2} u\right\|\right)\left(|x-y|+\left|t_{1}-t_{2}\right|^{1 / 2}\right)^{\gamma} .
$$

If $\frac{\epsilon}{2}>|x-y|^{2}+\left|t_{1}-t_{2}\right| \geq \frac{d_{x}^{2}}{4}$, it follows from Lemma 5.9 that

$$
\begin{aligned}
\left\|D^{2} u\left(x, t_{1}\right)-D^{2} u\left(y, t_{2}\right)\right\| & \leq\left\|D^{2} u\left(x, t_{1}\right)-D^{2} u\left(x_{0}, t_{1}\right)\right\|+\left\|D^{2} u\left(x_{0}, t_{1}\right)-D^{2} u\left(y, t_{2}\right)\right\| \\
& \leq C_{\epsilon, T}\left(\left|x-x_{0}\right|^{\beta / 2}+\left(\left|x_{0}-y\right|+\left|t_{1}-t_{2}\right|^{1 / 2}\right)^{\beta / 2}\right) \\
& \leq C\left(|x-y|+\left|t_{1}-t_{2}\right|^{1 / 2}\right)^{\beta / 2} \\
& \leq C\left(|x-y|+\left|t_{1}-t_{2}\right|^{1 / 2}\right)^{\gamma}
\end{aligned}
$$

where $C_{\epsilon, T}$ is the constant in Lemma 5.9, $x_{0} \in \partial \Omega$ satisfies $d_{x}=\left|x-x_{0}\right|$ and $C$ is universal.

\subsection{Higher regularity.}

Let $g \in C^{k+1, \alpha}(\bar{\Omega} \times[0, T))$, where $k \geq 0,0<\alpha<1$. Let $F$ be a function defined on $\operatorname{Mat}(N \times N, \mathbb{R}) \times \bar{\Omega} \times[0, T)$ such that $F(., x, t)$ is concave and satisfies (28). Assume that $F \in C^{k+2 ; k+1, \alpha}(\operatorname{Mat}(N \times N, \mathbb{R}) \times \bar{\Omega} \times[0, T))$, i.e., the derivaties $D_{r}^{i} D_{x}^{j} D_{t}^{l} F$ are continuous for all $|i| \leq k+2,|j|+2 l \leq k+1$ and satisfy

$$
\|F\|_{C^{k+2 ; k+1, \alpha}(\operatorname{Mat}(N \times N, \mathbb{R}) \times \bar{\Omega} \times[0, T))}=\sum_{|i| \leq k+2} \sup _{r \in \operatorname{Mat}(N \times N, \mathbb{R})}\left|D_{r}^{i} F(r, .)\right|_{C^{k+1, \alpha}(\bar{\Omega} \times[0, T))}<\infty .
$$

We consider the $C^{k+3, \alpha}$ regularity of a solution $u$ of the equation

$$
\dot{u}=F\left(D^{2} u, x, t\right)+g(x, t) .
$$

The following boundary estimates hold: 
Proposition 5.10. Let $x_{0} \in \partial \Omega, k \geq 0, r>0$ and $u \in C^{\infty}\left(\left(\Omega \cap B_{r}\left(x_{0}\right)\right) \times(0, T)\right) \cap$ $C^{k+2, \alpha}\left(\left(\Omega \cap B_{r}\left(x_{0}\right)\right) \times(0, T)\right)$ be a solution of

$$
\left\{\begin{array}{l}
\dot{u}=F\left(D^{2} u, x, t\right)+g(x, t) \text { on }\left(\Omega \cap B_{r}\left(x_{0}\right)\right) \times(0, T), \\
u=\varphi \text { on }\left(\partial \Omega \cap B_{r}\left(x_{0}\right)\right) \times(0, T)
\end{array}\right.
$$

where $\varphi \in C^{k+3, \alpha}\left(\bar{\Omega} \times(0, T)\right.$. Then there exists $r^{\prime} \in(0, r)$ depending on $r, \Omega$ such that $u \in C^{3+k, \alpha}\left(\left(\Omega \cap B_{r^{\prime}}\left(x_{0}\right)\right) \times\left(\epsilon, T^{\prime}\right)\right)$ for any $0<\epsilon<T^{\prime}<T$. Moreover

$$
\|u\|_{C^{k+3, \alpha}\left(\left(\Omega \cap B_{r^{\prime}}\left(x_{0}\right)\right) \times\left(\epsilon, T^{\prime}\right)\right)} \leq K
$$

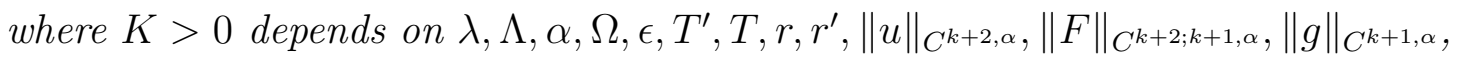
$\|\varphi\|_{C^{k+3, \alpha}}$.

This regularity is proved, for example, in [Lieb96] (or [GT83], [CC95] for the elliptic version). For the reader's convenience, we recall the arguments here.

Proof. Using a smooth diffeomorphism (as proof of Lemma 5.3), we can replace $\Omega \cap$ $B_{r}\left(x_{0}\right)$ by $B_{4}^{+}$and replace $\partial \Omega \cap B_{r}\left(x_{0}\right)$ by $\Gamma_{4}$. We need to show that $u \in C^{k+3, \alpha}\left(B_{1}^{+} \times\right.$ $\left.\left(\epsilon, T^{\prime}\right)\right)$.

Let $h>0$ be small and $e_{l}$ be the $l^{\text {th }}$ vector of the standard basis of $R^{N}, l<N$. We define

$$
\begin{aligned}
a_{i j}^{h}(x, t) & =\int_{0}^{1} \frac{\partial F}{\partial r_{i j}}\left(s D^{2} u\left(x+h e_{l}, t\right)+(1-s) D^{2} u(x, t), x+s h e_{l}, t\right) d s \\
g^{h}(x, t) & =\frac{g\left(x+h e_{l}, t\right)-g(x, t)}{h} \\
G^{h}(x, t) & =\int_{0}^{1} F_{l}\left(s D^{2} u\left(x+h e_{l}, t\right)+(1-s) D^{2} u(x, t), x+s h e_{l}, t\right) d s \\
\varphi^{h}(x, t) & =\frac{\varphi\left(x+h e_{l}, t\right)-\varphi(x, t)}{h} \\
v^{h}(x, t) & =\frac{u\left(x+h e_{l}, t\right)-u(x, t)}{h}
\end{aligned}
$$

For the convenience, we denote $Q_{a}=B_{a}^{+} \times(0, T)$ for any $a>0$. Then

$$
\left\|a_{i j}^{h}\right\|_{C^{k, \alpha}\left(Q_{2}\right)}+\left\|g^{h}\right\|_{C^{k, \alpha}\left(Q_{2}\right)}+\left\|G^{h}\right\|_{C^{k, \alpha}\left(Q_{2}\right)}+\left\|v^{h}\right\|_{C^{k+1, \alpha}\left(Q_{2}\right)}+\left\|\varphi^{h}\right\|_{C^{k+2, \alpha}\left(Q_{2}\right)}<A,
$$

where $A>0$ depends only on $\|u\|_{C^{k+2, \alpha}\left(Q_{4}\right)},\|F\|_{C^{k+2 ; k+1, \alpha}\left(Q_{4}\right)},\|g\|_{C^{k+1, \alpha}\left(Q_{4}\right)},\|\varphi\|_{C^{k+3, \alpha}\left(Q_{4}\right)}$. Moreover,

$$
\left\{\begin{array}{l}
\dot{v}^{h}=\sum a_{i j}^{h} v_{i j}^{h}+g^{h}+G^{h} \text { on } Q_{2}, \\
v^{h}=\varphi^{h} \text { on } \Gamma_{2} \times(0, T) .
\end{array}\right.
$$

If $k=0$, using a cutoff function and applying Schauder's global estimates ( [Fried83],page $65)$, we have

$$
\left\|v^{h}\right\|_{C^{k+2, \alpha}\left(B_{1}^{+} \times\left(\epsilon, T^{\prime}\right)\right)} \leq C,
$$

where $C>0$ depends on $A$ and $\epsilon, T^{\prime}$.

If $k>0$ and Proposition 5.10 is verified for $k-1$, then applying the case $k-1$, we also obtain (44).

It follows that $u_{l} \in C^{k+2, \alpha}\left(B_{1}^{+} \times\left(\epsilon, T^{\prime}\right)\right)$ with $\left\|u_{l}\right\|_{C^{k+2, \alpha}\left(B_{1}^{+} \times\left(\epsilon, T^{\prime}\right)\right)} \leq C$. 
By the same method, we can also show that $\|\dot{u}\|_{C^{k+1, \alpha}\left(B_{1}^{+} \times\left(\epsilon, T^{\prime}\right)\right)} \leq C$. It remains to prove $\left\|u_{N N N}\right\|_{C^{k, \alpha}\left(B_{1}^{+} \times\left(\epsilon, T^{\prime}\right)\right)} \leq C$. On $B_{1}^{+} \times\left(\epsilon, T^{\prime}\right)$, we have

$$
\dot{u}_{N}=\sum\left(\frac{\partial F}{\partial r_{i j}}\left(D^{2} u, x, t\right)\right) u_{i j N}+F_{N}\left(D^{2} u, x, t\right)+g_{N}(x, t) .
$$

Then

$$
u_{N N N}=\frac{1}{\partial F / \partial r_{N N}}\left(\dot{u}_{N}-\sum_{(i, j) \neq(N, N)} \frac{\partial F}{\partial r_{i j}} u_{i j N}-g_{N}\right) .
$$

Note that $\frac{\partial F}{\partial r_{N N}} \geq \lambda>0$. Hence, $u_{N N N} \in C^{k, \alpha}\left(B_{1}^{+} \times\left(\epsilon, T^{\prime}\right)\right)$ and $\left\|u_{N N N}\right\|_{C^{k, \alpha}\left(B_{1}^{+} \times\left(\epsilon, T^{\prime}\right)\right)}$ is bounded by a universal constant.

Using the method of the proof above, we also obtain the interior estimates

Proposition 5.11. Let $x_{0} \in \Omega$ and $0<r<d\left(x_{0}, \partial \Omega\right)$. Let $u \in C^{k+2, \alpha}\left(B_{r}\left(x_{0}\right) \times(0, T)\right)$ be a solution of

$$
\dot{u}=F\left(D^{2} u, x, t\right)+g(x, t) \text { on } B_{r}\left(x_{0}\right) .
$$

Then $u \in C^{k+3, \alpha}\left(B_{r / 2}\left(x_{0}\right) \times\left(\epsilon, T^{\prime}\right)\right)$ for any $0<\epsilon<T^{\prime}<T$. Moreover

$$
\|u\|_{C^{k+3, \alpha}\left(B_{r / 2}\left(x_{0}\right) \times\left(\epsilon, T^{\prime}\right)\right)} \leq C,
$$

where $C>0$ depends on $\lambda, \Lambda, \alpha, \epsilon, T^{\prime}, T, r,\|u\|_{C^{k+2, \alpha}},\|F\|_{C^{k+2 ; k+1, \alpha}},\|g\|_{C^{k+1, \alpha}}$.

Combining Proposition 5.10 and Proposition 5.11, we have the following

Proposition 5.12. Let $F, f, \varphi$ be functions defined as 5.2. Assume that $u \in C^{2, \alpha}(\Omega \times$ $(0, T))$ is a solution of

$$
\left\{\begin{array}{l}
\dot{u}=F\left(D^{2} u\right)+f(t, x, u) \text { on } \Omega \times(0, T), \\
u=\varphi \text { on } \partial \Omega \times(0, T) .
\end{array}\right.
$$

Then $u \in C^{\infty}(\bar{\Omega} \times(0, T))$.

\section{Proof of the MAIN THEOREM}

We recall the main theorem:

Theorem 6.1 (Main theorem). Let $\Omega$ be a bounded smooth strictly pseudoconvex domain of $\mathbb{C}^{n}$ and $T \in(0, \infty]$. Let $u_{0}$ be a bounded plurisubharmonic function defined on a neighbourhood $\tilde{\Omega}$ of $\bar{\Omega}$. Assume that $\varphi \in C^{\infty}(\bar{\Omega} \times[0, T))$ and $f \in C^{\infty}([0, T) \times \bar{\Omega} \times \mathbb{R})$ satisfying

(i) $f_{u} \leq 0$.

(ii) $\varphi(z, 0)=u_{0}(z)$ for $z \in \partial \Omega$.

Then there exists a unique function $u \in C^{\infty}(\bar{\Omega} \times(0, T))$ such that

$$
\begin{aligned}
& u(., t) \text { is a strictly plurisubharmonic function on } \Omega, \forall t \in(0, T), \\
& \qquad \begin{array}{c}
\dot{u}=\log \operatorname{det}\left(u_{\alpha \bar{\beta}}\right)+f(t, z, u) \text { on } \Omega \times(0, T), \\
u=\varphi \text { on } \partial \Omega \times(0, T),
\end{array}
\end{aligned}
$$




$$
\lim _{t \rightarrow 0} u(z, t)=u_{0}(z) \forall z \in \bar{\Omega} .
$$

Moreover, $u \in L^{\infty}\left(\bar{\Omega} \times\left[0, T^{\prime}\right)\right)$ for any $0<T^{\prime}<T$, and $u(., t)$ also converges to $u_{0}$ in capacity when $t \rightarrow 0$.

If $u_{0} \in C(\tilde{\Omega})$ then $u \in C(\bar{\Omega} \times[0, T))$.

Proof. Replacing $T$ by $0<T^{\prime}<T$, we can assume that $T<\infty$ and there exists $C_{\varphi}$ such that

$$
\|\varphi\|_{C^{4}(\Omega \times(0, T))} \leq C_{\varphi} .
$$

We can also assume that $\|f\|_{C^{2}([0, T) \times \bar{\Omega} \times[-M, M])}<\infty$ for any $M>0$.

\section{Existence of a solution.}

Using the convolution of $u_{0}+\frac{|z|^{2}}{m}$ with smooth kernels, we can take $u_{0, m} \in C^{\infty}(\bar{\Omega})$ such that

$$
\begin{gathered}
u_{0, m} \searrow u_{0}, \\
d d^{c} u_{0, m} \geq \frac{1}{m} d d^{c}|z|^{2} .
\end{gathered}
$$

Note that $\left.u_{0}\right|_{\partial \Omega}$ is continuous. Then

$$
\delta_{m}=\sup _{z \in \partial \Omega}\left(u_{0, m}(z)-u_{0}(z)\right) \stackrel{m \rightarrow \infty}{\longrightarrow} 0 .
$$

We define $g_{m} \in C^{\infty}(\bar{\Omega})$ and $\varphi_{m} \in C^{\infty}(\bar{\Omega} \times[0, T))$ by

$$
\begin{gathered}
g_{m}=-\log \operatorname{det}\left(u_{0, m}\right)_{\alpha \bar{\beta}}+f\left(0, z, u_{0, m}\right), \\
\varphi_{m}=\zeta\left(\frac{t}{\epsilon_{m}}\right)\left(t g_{m}+u_{0, m}\right)+\left(1-\zeta\left(\frac{t}{\epsilon_{m}}\right)\right) \varphi
\end{gathered}
$$

where $\zeta$ is a smooth funtion on $\mathbb{R}$ such that $\zeta$ is decreasing, $\left.\zeta\right|_{(-\infty, 1]}=1$ and $\left.\zeta\right|_{[2, \infty)}=0$. $\epsilon_{m}>0$ are chosen such that the sequences $\left\{\epsilon_{m}\right\},\left\{\epsilon_{m} \sup \left|g_{m}\right|\right\}$ are decreasing to 0 and $\zeta\left(\frac{t}{\epsilon_{m}}\right)\left(u_{0, m}(z)-\varphi(z, t)\right) \geq 0$ for any $m$.

Then $\varphi_{m}$ converges pointwise to $\varphi$ on $\partial \Omega \times[0, T)$ and for any $0<\epsilon<T$, there exists $m_{\epsilon}>0$ such that $\left.\varphi_{m}\right|_{\bar{\Omega} \times(\epsilon, T)}=\left.\varphi\right|_{\bar{\Omega} \times(\epsilon, T)}, \forall m>m_{\epsilon}$.

Moveover,

$$
\begin{gathered}
\varphi_{m}(z, 0)=u_{0, m}(z), \\
\dot{\varphi}_{m}=\log \operatorname{det}\left(u_{0, m}\right)_{\alpha \bar{\beta}}+f\left(t, z, u_{0, m}\right),
\end{gathered}
$$

where $(z, t) \in \partial \Omega \times\{0\}$.

By the theorem of Hou-Li, there exists $u_{m} \in C^{\infty}(\Omega \times(0, T)) \cap C^{2 ; 1}(\bar{\Omega} \times[0, T))$ satisfying

$$
\begin{cases}\dot{u}_{m}=\log \operatorname{det}\left(u_{m}\right)_{\alpha \bar{\beta}}+f\left(t, z, u_{m}\right) & \text { on } \Omega \times(0, T), \\ u_{m}=\varphi_{m} & \text { on } \partial \Omega \times[0, T), \\ u_{m}=u_{0, m} & \text { on } \bar{\Omega} \times\{0\} .\end{cases}
$$

Applying Corollary 2.5 for $u_{1}$ and $u_{m}$, we see that the functions $u_{m}$ are uniformly bounded by a constant $C_{u}>0$. Then we can assume that $\|f\|_{C^{2}((0, T) \times \Omega \times \mathbb{R})} \leq C_{f}$. Applying Theorem 1.1 on $\Omega \times\left(\frac{\epsilon}{2}, T\right)$, we obtain

$$
\left\|u_{m}\right\|_{C^{2}(\Omega \times(\epsilon, T))} \leq C,
$$


where $C=C\left(\epsilon, T, \Omega, C_{f}, C_{\varphi}, C_{u}\right), m$ is large enough.

It follows from the $C^{2, \alpha}$ estimates in Section 5 that for any $0<\epsilon<T^{\prime}<T$, there exist $M=M\left(\epsilon, T^{\prime}, C, \Omega, C_{\varphi}, C_{f}\right)$ and $0<\gamma<1$ such that

$$
\left\|u_{m}\right\|_{C^{2, \gamma}(\bar{\Omega} \times(\epsilon, T))} \leq M \text {. }
$$

By Ascoli's theorem, there exists $u \in C^{2, \gamma / 2}(\bar{\Omega} \times(0, T))$ such that

$$
u_{m_{k}} \stackrel{C^{2, \gamma / 2}(\bar{\Omega} \times(\epsilon, T))}{\longrightarrow} u \text {. }
$$

Thus $u$ satisfies (47), (48) and (49). By Proposition 5.12 we have $u \in C^{\infty}(\bar{\Omega} \times(0, T))$. Clearly, $u$ is bounded. We need to show the convergence of $u(., t)$ when $t \rightarrow 0$.

Step 1: $\liminf _{t \rightarrow 0} u(z, t) \geq u_{0}(z)$.

By (54), there exists a subsequence of $\left(u_{m}\right)$, also denoted by $\left(u_{m}\right)$, which converges pointwise to $u$ on $\bar{\Omega} \times(0, T)$.

For any $a>0$, there exists $A>0$ such that $\forall m>0, v_{m}=u_{0, m}+a \rho-A t$ satisfies

$$
\left\{\begin{array}{l}
\dot{v}_{m} \leq \log \operatorname{det}\left(v_{m}\right)_{\alpha \bar{\beta}}+f\left(t, z, v_{m}\right), \\
\left.v_{m}\right|_{\partial_{P}(\Omega \times(0, T))} \leq\left. u_{m}\right|_{\partial_{P}(\Omega \times(0, T))}+\epsilon_{m} \sup \left|g_{m}\right|+\delta_{m},
\end{array}\right.
$$

where $\rho \in C^{\infty}(\bar{\Omega})$ is a non-positive strictly plurisubharmonic function on $\Omega$.

It follows from Corollary 2.5 that

$$
v_{m} \leq u_{m}+\epsilon_{m} \sup \left|g_{m}\right|+\delta_{m} .
$$

Hence

$$
u(z, t) \geq \lim _{m \rightarrow \infty}\left(v_{m}(z, t)-\epsilon_{m} \sup \left|g_{m}\right|-\delta_{m}\right)=u_{0}(z)+a \rho(z)-A t .
$$

Then we have

$$
\liminf _{t \rightarrow 0} u(z, t) \geq u_{0}(z)+a \rho(z)
$$

When $a \rightarrow 0$, we obtain

$$
\liminf _{t \rightarrow 0} u(z, t) \geq u_{0}(z)
$$

Step 2: $\limsup _{t \rightarrow 0} u(z, t) \leq u_{0}(z)$.

Let $\epsilon>0$. Assume that $m_{0}>0$ satisfies $\epsilon_{m_{0}} \sup \left|g_{m_{0}}\right| \leq \epsilon$.

For any $m>k>m_{0}$, we have

$$
\begin{aligned}
u_{0, m}-u_{0, k} & \leq 0 \\
\varphi_{m}-\varphi_{k} & =\zeta\left(\frac{t}{\epsilon_{m}}\right)\left(u_{0, m}-\varphi\right)-\zeta\left(\frac{t}{\epsilon_{k}}\right)\left(u_{0, k}-\varphi\right) \\
& +t g_{m} \zeta\left(\frac{t}{\epsilon_{m}}\right)-t g_{k} \zeta\left(\frac{t}{\epsilon_{k}}\right) \\
& \leq \zeta\left(\frac{t}{\epsilon_{k}}\right)\left(u_{0, m}-\varphi\right)-\zeta\left(\frac{t}{\epsilon_{k}}\right)\left(u_{0, k}-\varphi\right)+2 \epsilon \\
& \leq \zeta\left(\frac{t}{\epsilon_{k}}\right)\left(u_{0, m}-u_{0, k}\right)+2 \epsilon \\
& \leq 2 \epsilon .
\end{aligned}
$$

It follows Corollary 2.5 that

$$
u_{m} \leq u_{k}+2 \epsilon
$$

Hence

$$
u(z, t)=\lim _{m \rightarrow \infty} u_{m}(z, t) \leq u_{k}(z, t)+2 \epsilon
$$


Then we have

$$
\limsup _{t \rightarrow 0} u(z, t) \leq u_{0, k}(z)+2 \epsilon
$$

When $k \rightarrow \infty$ and $\epsilon \rightarrow 0$, we obtain

$$
\limsup _{t \rightarrow 0} u(z, t) \leq u_{0}(z)
$$

Combining (57) and (59), we obtain (50).

Step 3: Convergence in capacity.

The bounded plurisubharmonic function $u_{0}$ is continuous outside sets of arbitrarily small capacity. Then the convergence in capacity is implied by (56), (58) and Hartogs lemma (Lemma 90 of [Ber13]) .

If $u_{0} \in C(\tilde{\Omega})$ then $u_{0, m}$ and $\varphi_{m}$ converge uniformly, respectively, to $u_{0}$ and $\varphi$. It follows Corollary 2.5 that $u_{m}$ converges uniformly to $u$. So $u$ is continuous on $\bar{\Omega} \times[0, T)$.

\section{Uniqueness of the solution.}

Let $u, v \in C^{\infty}(\bar{\Omega} \times(0, T))$ be functions satisfying (47), (48), (49), (150). Let $\epsilon>0$. We need to show that $u \leq v+(t+3) \epsilon$.

Step 1. $\exists A>0, v(z, t) \geq u_{0}(z)-\epsilon-$ At.

For $m>0$, we denote $v_{m}(z, t)=v\left(z, t+\frac{1}{m}\right)$. Then $v_{m}$ is the solution of

$$
\left\{\begin{array}{l}
\dot{v}_{m}=\log \operatorname{det}\left(v_{m}\right)_{\alpha \bar{\beta}}+f\left(t+\frac{1}{m}, z, v_{m}\right) \text { on } \Omega \times\left(0, T-\frac{1}{m}\right), \\
v_{m}(z, t)=\varphi\left(z, t+\frac{1}{m}\right) \text { on } \partial \Omega \times\left(0, T-\frac{1}{m}\right) .
\end{array}\right.
$$

Let $\rho \in C^{\infty}(\bar{\Omega})$ be a non-positive strictly plurisubharmonic function on $\Omega$ such that inf $\rho=-1$. Then there exists $A>0$ depending only on $\epsilon, \rho,\|\varphi\|_{C^{1}}, \sup f(t, z, \sup \varphi)$ such that

$$
\left\{\begin{array}{l}
\dot{w}_{m} \leq \log \operatorname{det}\left(w_{m}\right)_{\alpha \bar{\beta}}+f\left(t+\frac{1}{m}, z, w_{m}\right) \text { on } \Omega \times\left(0, T-\frac{1}{m}\right) \\
w_{m}(z, t) \leq \varphi\left(z, t+\frac{1}{m}\right) \text { on } \partial \Omega \times\left(0, T-\frac{1}{m}\right)
\end{array}\right.
$$

where $w_{m}=v\left(z, \frac{1}{m}\right)+\epsilon \rho-A t$.

Applying Corollary 2.5, we have $v_{m} \geq w_{m}$. When $m \rightarrow \infty$, we obtain

$$
v(z, t) \geq u_{0}(z)+\epsilon \rho(z)-A t \geq u_{0}(z)-\epsilon-A t .
$$

Step 2. $\exists m_{0}>0, \forall m>m_{0}, \exists k_{m}>m, v\left(z, \frac{1}{m}\right) \geq-3 \epsilon+u\left(z, \frac{1}{k_{m}}\right)$.

Step 1 implies that $v$ is bounded. Then we can assume that $\|f\|_{C^{2}([0, T) \times \bar{\Omega} \times \mathbb{R})}<\infty$.

By step 1, we have

$$
v\left(z, \frac{1}{m}\right)+\epsilon+\frac{A}{m} \geq u_{0}(z)=\lim _{t \rightarrow 0} u(z, t) .
$$

Applying Hartogs lemma, for any $K \Subset \Omega$ there exists $k_{m, K}>m$ such that

$$
u\left(z, \frac{1}{k_{m, K}}\right) \leq v\left(z, \frac{1}{m}\right)+2 \epsilon+\frac{A}{m} \forall z \in K .
$$

Let $m_{0} \geq \frac{1}{\epsilon} \max \left\{1, A,\|f\|_{C^{2}},\|h\|_{C^{2}}\right\}$, where $h \in C^{\infty}(\bar{\Omega} \times[0, T))$ is a spatial harmonic function such that $\left.h\right|_{\partial \Omega \times(0, T)}=\left.\varphi\right|_{\partial \Omega \times(0, T)}$.

For any $m>m_{0}$, let $K=K_{m} \Subset \Omega$ such that

$$
v\left(z, \frac{1}{m}\right)+\epsilon \geq h\left(z, \frac{1}{m}\right) \forall z \in \Omega \backslash K .
$$


Let $k_{m}=k_{m, K_{m}}$. Then

$$
v\left(z, \frac{1}{m}\right) \geq-2 \epsilon+h\left(z, \frac{1}{k_{m}}\right) \geq-2 \epsilon+u\left(z, \frac{1}{k_{m}}\right) \forall z \in \Omega \backslash K .
$$

Combining (62) and (63), we obtain

$$
v\left(z, \frac{1}{m}\right) \geq-3 \epsilon+u\left(z, \frac{1}{k_{m}}\right) \forall z \in \Omega .
$$

Step 3. Conclusion.

Let $u_{m}(z, t)=u\left(z, t+\frac{1}{k_{m}}\right)-\epsilon t$. For $m>m_{0}$, we have

$$
\left\{\begin{array}{l}
\dot{v}_{m}=\log \operatorname{det}\left(v_{m}\right)_{\alpha \bar{\beta}}+f\left(t+\frac{1}{m}, z, v_{m}\right) \geq \log \operatorname{det}\left(v_{m}\right)_{\alpha \bar{\beta}}+f\left(t+\frac{1}{k_{m}}, z, v_{m}\right)-\epsilon, \\
\dot{u}_{m} \leq \log \operatorname{det}\left(u_{m}\right)_{\alpha \bar{\beta}}+f\left(t+\frac{1}{k_{m}}, z, u_{m}\right)-\epsilon
\end{array}\right.
$$

Applying Corollary 2.5, we have

$$
\left(u_{m}-v_{m}\right) \leq \sup _{\partial_{P}\left(\Omega \times\left(0, T-\frac{1}{m}\right)\right)}\left(u_{m}-v_{m}\right) \leq 3 \epsilon
$$

When $m \rightarrow \infty$, we have

$$
u(z, t)-v(z, t)-\epsilon t=\lim _{m \rightarrow \infty}\left(u_{m}(z, t)-v_{m}(z, t)\right) \leq 3 \epsilon .
$$

When $\epsilon \rightarrow 0$, we obtain

$$
u(z, t) \leq v(z, t) .
$$

Since the roles of $u$ and $v$ are symmetric, $v(z, t) \leq u(z, t)$. Then $u=v$.

\section{FurTher DiRECTIONS}

In this section, we discuss further questions in the same general directions as our result. On compact Kähler manifolds, the corresponding problem was solved in the case where $f=0$ and $u_{0}$ has zero Lelong numbers. In that case, there exists a solution $u$ satisfying $u(., t) \rightarrow u_{0}$ in $L^{1}$ (see GZ13]), and the solution is unique (see [DL14]). It is natural to ask whether the same result holds for a domain in $\mathbb{C}^{n}$. Let us state our conjecture

Conjecture 7.1. If we replace the condition " $u_{0} \in L^{\infty}(\tilde{\Omega})$ " in Theorem 6.1 by the condition " $u_{0}$ has zero Lelong numbers" then there exists a unique function $u \in C^{\infty}(\bar{\Omega} \times$ $(0, T)$ ) satisfying (47), (48), (49) such that $u(., t) \rightarrow u_{0}$ in $L^{1}(\Omega)$.

The case where $u_{0}$ has positive Lelong numbers is another problem. It was also considered and solved in the case compact Kähler manifold by [GZ13] and [DL14]. It is the motivation of the second direction: the case of domain in $\mathbb{C}^{n}$ and $u_{0}$ has positive Lelong numbers.

There is another question: What is the behavior when we replace the condition " $u_{0} \in P S H(\tilde{\Omega})$ " in Theorem 6.1 by the condition " $u_{0} \in P S H(\Omega)$ "? In order to prove Theorem 6.1, we construct plurisubharmonic functions $u_{0, m}$ which converge to $u_{0}$. This step is easy if we suppose that $u_{0} \in P S H(\tilde{\Omega})$. If we only suppose that $" u_{0} \in P S H(\Omega)$ and $\lim _{z \rightarrow z_{0} \in \partial \Omega} u_{0}(z)=\varphi\left(z_{0}\right)$ ", maybe this step is still realizable but more difficult. We give a provisional result in this direction. 
Proposition 7.2. Let $\Omega$ be a bounded smooth strictly pseudoconvex domain of $\mathbb{C}^{n}$ and $T \in(0, \infty]$. Let $u_{0}$ be a continuous plurisubharmonic function on $\Omega$ such that $u_{0}$ is smooth on $\bar{\Omega} \backslash \mathcal{K}$, where $\mathcal{K} \Subset \Omega$. Assume that $\varphi, f$ are functions satisfying the conditions of Theorem 6.1. Then there exists a unique function $u \in C^{\infty}(\bar{\Omega} \times(0, T)) \cap C(\bar{\Omega} \times[0, T))$ satisfying (47), (48), (49) and $u(., 0)=u_{0}$.

Proof sketch. Let $\rho, \zeta$ be the functions defined in the proof of Theorem 6.1. Let $\psi$ be a smooth function in $\Omega$ and $\phi$ be a smooth function on $\mathbb{R}$ satisfying

- $0 \leq \psi \leq 1,\left.\psi\right|_{U_{1}}=1,\left.\psi\right|_{\Omega \backslash U_{2}}=0$, where $\mathcal{K} \Subset U_{1} \Subset U_{2} \Subset \Omega$.

- $\phi$ is convex and increasing, $\left.\phi\right|_{(-\infty,-3)}=-2,\left.\phi\right|_{(-1, \infty)}=I d$.

Using convolutions of $u_{0}+\frac{\rho}{m}$, we can find $\tilde{u}_{0, m} \in C^{\infty}\left(U_{2}\right)$ such that $\tilde{u}_{0, m}$ and $\psi \tilde{u}_{0, m}+$ $(1-\psi)\left(u_{0}+\frac{\rho}{m}\right)$ are strictly plurisubharmonic functions.

We define $u_{0, m} \in C^{\infty}(\bar{\Omega}), g_{m} \in C^{\infty}(\bar{\Omega} \backslash \mathcal{K}), \varphi_{m} \in C^{\infty}(\bar{\Omega} \times[0, T))$ by

$$
\begin{gathered}
u_{0, m}=\psi \tilde{u}_{0, m}+(1-\psi)\left(u_{0}+\frac{\rho}{m}\right)+\frac{1}{m} \phi \circ(m \rho), \\
g_{m}=-\left.\dot{\varphi}\right|_{t=0}+\log \operatorname{det}\left(u_{0}+\frac{m+1}{m} \rho\right)_{\alpha \bar{\beta}}+f\left(t, z, u_{0}+\frac{m+1}{m} \rho\right), \\
\varphi_{m}=(1-\psi)\left(t \zeta(m t) g_{m}+u_{0}+\frac{m+1}{m} \rho+\int_{0}^{t} \dot{\varphi}\right) .
\end{gathered}
$$

Repeating the techniques in the proof of Theorem 6.1, we show that there exists a unique function $u \in C^{\infty}(\bar{\Omega} \times(0, T)) \cap C(\bar{\Omega} \times[0, T))$ satisfying (47), (48), (49)) such that $\left.u\right|_{t=0}=u_{0}$.

\section{REFERENCES}

[Ber13] F. BERTELOOT: Bifurcation currents in holomorphic families of rational maps. Pluripotential theory 1-93, Lecture Notes in Math., 2075, Springer, Heidelberg, 2013.

[BG13] S. BOUCKSOM, V. GUEDJ: Regularizing properties of the Kähler-Ricci flow. An introduction to the Kähler-Ricci flow,189-237, Lecture Notes in Math., 2086, Springer, Cham, 2013.

[Blo08] Z. BLOCKI: A gradient estimate in the Calabi-Yau theorem. Math. Ann. 344 (2009), 317-327.

[Bou11] S. BOUCKSOM: Monge-Ampère equations on complex manifolds with boundary.Complex Monge-Ampère equations and geodesics in the space of Khler metrics, 257-282, Lecture Notes in Math., 2038, Springer, Heidelberg, 2012.

[Cao85] H-D.CAO: Deformation of Kähler metrics to Kähler-Einstein metrics on compact Kähler manifolds. Invent. Math. 81 (1985), no. 2, 359-372.

[CC95] L. CAFFARELLI, X. CABRE: Fully nonlinear elliptic equations, Colloquium publications 43, American Mathematical Society, Providence, RI, 1995.

[CKNS85] L. CAFFARELLI, J. KOHN, L. NIRENBERG, J. SPRUCK: The Dirichlet problem for nonlinear second-order elliptic equations. II. Complex Monge-Ampère, and uniform elliptic, equations. C.P.A.M. 38 (1985) no. 2, 209-252.

[DL14] E. DI NEZZA, H.-C. LU: Uniqueness and short time regularity of the weak Kähler-Ricci flow. arXiv:math.CV/14117958]

[EGZ14] P. EYSSIDIEUX, V. GUEDJ, A. ZERIAHI: Weak solutions to degenerate complex MongeAmpère flows $I$. arXiv:math.CV/14072494v1]

[Fried83] A. FRIEDMAN: Partial differential equations of Parabolic type, Krieger, Malabar, 1983. 
[GT83] D. GILBARG, N. TRUDINGER:Elliptic partial differential equations of second order. Second edition. Grundlehren der Mathematischen Wissenschaften 224.Springer-Verlag, Berlin, 1983. xiii+513 pp.

[Gua98] B. GUAN:The Dirichlet problem for complex Monge-Ampère equations and regularity of the pluri-complex Green function. Comm.Anal. Geom. 6 (1998), no. 4, 687-703.

[GZ13] V. GUEDJ, A. ZERIAHI: Regularizing properties of the twisted Kähler - Ricci flow. arXiv:math.CV/13064089v1]

[HL10] Z. HOU, Q. LI: Energy functionals and complex Monge-Ampère equations. J. Inst. Math. Jussieu 9 (2010) no.3, 463-476.

[IS13] C. IMBERT, L. SILVESTRE: An introduction to fully nonlinear parabolic equations. An introduction to the Kähler-Ricci flow, 7-88, Lecture Notes in Math., 2086, Springer, Cham, 2013.

[Kryl96] N.V. KRYLOV: Lectures on elliptic and parabolic equations in Hölder spaces, Graduate Studies in Mathematics, vol. 12 (American Mathematical Society).

[Lieb96] G.M. LIEBERMAN: Second order parabolic differential equations(World Scientific, River Edge, 1996).

[PS05] D. H. PHONG, J. STURM: On the Kähler-Ricci flow on complex surfaces. Pure Appl. Math. Q. 1 (2005), no. 2, part 1, 405-413.

[Siu87] Y.T. SIU: Lectures on Hermitian-Einstein metrics for stable bundles and Kähler-Einstein metrics. DMV Seminar, 8. Birkhäuser Verlag, Basel, 1987.

[ST07] J. SONG, G. TIAN: The Kähler-Ricci flow on surfaces of positive Kodaira dimension. Invent. Math. 170 (2007), no. 3, 609-653.

[Tos10] V. TOSATTI: Kähler-Ricci flow on stable Fano manifolds.J. Reine Angew. Math. 640 (2010), $67-84$.

[Yau78] S.T. YAU: On the Ricci curvature of a compact Kähler manifold and the complex MongeAmpère equation. I. Comm. Pure Appl. Math. 31 (1978), no. 3, 339-411.

[Zha09] Z. ZHANG: Scalar curvature bound for Kähler-Ricci flows over minimal manifolds of general type. Int. Math. Res. Not. IMRN 2009, no. 20, 3901-3912.

E-mail address: hoangson.do.vn@gmail.com 